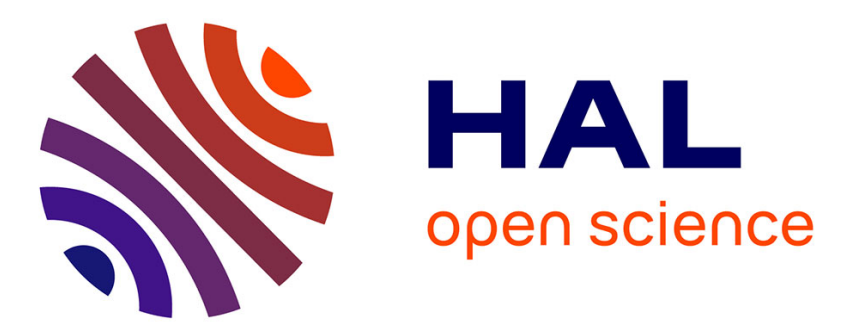

\title{
High order HDG method and domain decomposition solvers for frequency-domain electromagnetics
}

\author{
Emmanuel Agullo, Luc Giraud, Alexis Gobé, Matthieu Kuhn, Stephane
}

Lanteri, Ludovic Moya

\section{- To cite this version:}

Emmanuel Agullo, Luc Giraud, Alexis Gobé, Matthieu Kuhn, Stephane Lanteri, et al.. High order HDG method and domain decomposition solvers for frequency-domain electromagnetics. International Journal of Numerical Modelling: Electronic Networks, Devices and Fields, 2019, 10.1002/jnm.2678 . hal-02327982

\section{HAL Id: hal-02327982 \\ https://hal.inria.fr/hal-02327982}

Submitted on 12 Nov 2019

HAL is a multi-disciplinary open access archive for the deposit and dissemination of scientific research documents, whether they are published or not. The documents may come from teaching and research institutions in France or abroad, or from public or private research centers.
L'archive ouverte pluridisciplinaire HAL, est destinée au dépôt et à la diffusion de documents scientifiques de niveau recherche, publiés ou non, émanant des établissements d'enseignement et de recherche français ou étrangers, des laboratoires publics ou privés. 


\title{
High order HDG method and domain decomposition solvers for frequency-domain electromagnetics
}

\author{
Emmanuel Agullo ${ }^{1}$ | Luc Giraud $^{1}$ | Alexis Gobé ${ }^{2}$ | Matthieu Kuhn ${ }^{1}$ | Stéphane \\ Lanteri $^{* 2}$ | Ludovic Moya ${ }^{2}$
}

${ }^{1}$ Hiepacs project-team, Inria Bordeaux Sud-Ouest France

${ }^{2}$ Nachos project-team, Inria Sophia Antipolis Méditerranée France

Correspondence

*Corresponding author name, Inria Sophia Antipolis - Méditerranée, France. Email:

Stephane.Lanteri@inria.fr

Present Address

\begin{abstract}
Summary
This work is concerned with the numerical treatment of the system of three-dimensional frequency-domain (or time-harmonic) Maxwell equations using a high order hybridizable discontinuous Galerkin (HDG) approximation method combined to domain decomposition (DD) based hybrid iterative-direct parallel solution strategies. The proposed HDG method preserves the advantages of classical DG methods previously introduced for the time-domain Maxwell equations, in particular in terms of accuracy and flexibility with regards to the discretization of complex geometrical features, while keeping the computational efficiency at the level of the reference edge element based finite element formulation widely adopted for the considered PDE system. We study in details the computational performances of the resulting DD solvers in particular in terms of scalability metrics by considering both a model test problem and more realistic large-scale simulations performed on high performance computing systems consisting of networked multicore nodes.
\end{abstract}

\section{1 | INTRODUCTION}

When dealing with the simulation of the propagation of time-harmonic electromagnetic waves in heterogeneous media, the finite

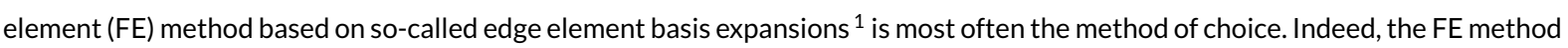
is nowadays implemented in major electromagnetics commercial simulation software such as HFS\$1 ${ }^{1}$ or COMSOL Multiphysics $2^{2}$ to cite a few. In the recent years, Discontinuous Galerkin (DG) methods have ben extensively studied for the numerical solution of the time-domain Maxwell equations. Thanks to the discontinuity of the approximation, this kind of methods has many advantages, such as adaptivity to complex geometries through the use of unstructured possibly non-conforming meshes, easily obtained high order accuracy, hp-adaptivity and improved parallel efficiency.

For the numerical treatment of the frequency-domain Maxwell equations, classical DG methods can also be considered 2 -3. ever, such DG formulations are highly expensive, especially for the discretization of three-dimensional problems, because they lead

\footnotetext{
${ }^{1}$ https://www.ansys.com/products/electronics/ansys-hfss

${ }^{2}$ https://www.comsol.com
} 
to a large sparse and indefinite linear system of equations coupling all the degrees of freedom of the unknown physical fields. As a consequence, the number of globally coupled DG degrees of freedom is much greater than the number required by conforming finite element methods for the same accuracy. Different attempts have been made in the recent past to improve the efficiency of DG methods applied to steady-like problems and one promising strategy has been proposed by Cockburn et al. ${ }^{4}$ in the form of so-called hybridizable discontinuous Galerkin (HDG) formulations.

HDG methods introduce an additional hybrid variable on the faces of the elements, on which the definition of the local (elementwise) solutions is based. A so-called conservativity condition is imposed on the numerical trace, whose definition involved the hybrid variable, at the interface between neighboring elements 456 . As a result, HDG methods produce a linear system in terms of the DOFs of the additional hybrid variable only. In this way, the number of globally coupled DOFs is reduced. The local values of the electromagnetic fields can be obtained by solving local problems element-by-element.

In the follow-up of these seminal works, most of the recent contributions on HDG methods for the frequency-domain Maxwell equations are concerned with theoretical questions. A theoretical assesmment of a HDG method for the three-dimensional timeharmonic Maxwell in the case of high wave number is realized in 7 . The authors prove that the HDG method is absolutely stable for all wave numbers in the sense that no mesh constraint is required for the stability. $\ln 8$ the authors present and analyze a HDG method for the time-harmonic Maxwell equations in curl-curl form, in which the divergence-free condition is enforced on the electric field. The proposed HDG method is shown to be absolutely stable for the indefinite time-harmonic Maxwell equations with high wave numbers.

The goal of this paper is to report on further developments of the HDG method proposed in 9 aiming at improving its accuracy and scalability for the simulation of large-scale three-dimensional problems. As such, this paper does not present some new HDG formulation or theoretical results on the formulation initially proposed in ${ }^{9}$, and is rather meant to offer an updated picture of the development of this method towards its adoption for large scale simulations. Section 2 defines the considered boundary value problem for the three-dimensiobal time-harmonic Maxwell equations and introduces some notations. Section 3 presents the principles and general formulation of the HDG method. The implementation of the HDG method is the subject of section 4 Though the HDG method results in a smaller linear system than the one associated with a classical upwind flux-based DG method, the size of this system is often too large to be solved by a sparse direct solver as soon as one consider realistic three-dimensional problems. In addition, for very large-scale propagation problems, exploiting a multi-processor system is a mandatory path to reduce the time to solution and have access to the required memory capacity. In section 5 . we briefly discuss about the solution strategies that we have considered in this work, in particular the PDE-based and algebraic domain decomposition solvers that have been initially developed in other contexts. Numerical examples are given in section 6 with two objectives in mind: first, using a simple (model) problem, the proposed DD-HDG solution strategy is validated and its convergence properties are assessed; second, by considering more complex problems, we study the overall efficiency of the DD-HDG solution strategy. Finally, we draw some conclusions and state future works in section 7

\section{2 | PROBLEM STATEMENT AND NOTATIONS}

\section{1 | Boundary value problem}

The system of $3 \mathrm{~d}$ time-harmonic Maxwell equations is considered

$$
\left\{\begin{array}{l}
\mathrm{i} \omega \varepsilon_{\mathrm{r}} \mathbf{E}-\operatorname{curl} \mathbf{H}=-\mathbf{J}, \text { in } \Omega, \\
\mathrm{i} \omega \mu_{\mathrm{r}} \mathbf{H}+\operatorname{curl} \mathbf{E}=0, \text { in } \Omega,
\end{array}\right.
$$

where $\mathrm{i}$ is the imaginary unit, $\omega$ is the angular frequency, $\varepsilon_{\mathrm{r}}$ and $\mu_{\mathrm{r}}$ are the relative permittivity and permeability, $\mathbf{E}$ and $\mathbf{H}$ are the electric and magnetic fields, $\mathbf{J}$ is a known current source. The boundary of the computational domain $\Omega$ is $\partial \Omega=\Gamma_{m} \cup \Gamma_{a}\left(\Gamma_{m} \cap \Gamma_{a}=\emptyset\right)$ 
on which we impose the following boundary conditions

$$
\left\{\begin{aligned}
\mathbf{n} \times \mathbf{E} & =0, \text { on } \Gamma_{\mathrm{m}}, \\
\mathbf{n} \times \mathbf{E}+\mathbf{n} \times(\mathbf{n} \times \mathbf{H}) & =\mathbf{n} \times \mathbf{E}^{\text {inc }}+\mathbf{n} \times\left(\mathbf{n} \times \mathbf{H}^{\text {inc }}\right)=\mathbf{g}^{\text {inc }}, \text { on } \Gamma_{\mathrm{a}},
\end{aligned}\right.
$$

where $\mathbf{n}$ is the outward unit normal vector and $\left(\mathbf{E}^{\text {inc }}, \mathbf{H}^{\text {inc }}\right)$ is a given incident electromagnetic wave. The boundary condition on $\Gamma_{m}$ indicates a metallic boundary condition (also called perfect electric conductor condition), while the second relation on $\Gamma_{\mathrm{a}}$ states a Silver-Müller condition (first order absorbing boundary condition). For sake of simplicity, we omit the volume source term $\mathbf{J}$ in what follows but it can be straightforwardly added.

\section{2 | Notations}

We consider a simplicial mesh $\mathcal{T}_{\mathrm{h}}$ consisting of tetrahedral element $\mathrm{K}$ of the computational domain $\Omega$. We denote by $\mathcal{F}_{\mathrm{h}}$ the union of all interior interfaces of $\mathcal{T}_{\mathrm{h}}$, by $\mathcal{F}_{\mathrm{h}}^{\mathrm{B}}$ the union of all boundary interfaces of $\mathcal{T}_{\mathrm{h}}$, and by $\mathcal{F}_{\mathrm{h}}=\mathcal{F}_{\mathrm{h}}^{1} \cup \mathcal{F}_{\mathrm{h}}^{\mathrm{b}}$. Note that $\partial \mathcal{T}_{\mathrm{h}}$ is the set of all triangular faces forming the boundary $\partial \mathrm{K}$, for all elements $\mathrm{K}$ of $\mathcal{T}_{\mathrm{h}}$. Consequently, an interior face shared by two neighboring elements appears twice in this set $\partial \mathcal{T}_{\mathrm{h}}$, while it appears once in the set $\mathcal{F}_{\mathrm{h}}$. For an interface $\mathrm{F}=\overline{\mathrm{K}}^{+} \cap \overline{\mathrm{K}}^{-} \in \mathcal{F}_{\mathrm{h}}^{\prime}$, let $\mathbf{v}^{ \pm}$be the trace of $\mathbf{v}$ on $\mathrm{F}$ from $\mathrm{K}^{ \pm}$. We define on this face mean values $\{\cdot\}$ and jumps $\llbracket \cdot \rrbracket$

$$
\{\mathbf{v}\}_{F}=\frac{1}{2}\left(\mathbf{v}^{+}+\mathbf{v}^{-}\right) \text {, and } \llbracket \mathbf{v} \rrbracket_{F}=\mathbf{n}^{+} \times \mathbf{v}^{+}+\mathbf{n}^{-} \times \mathbf{v}^{-},
$$

where $\mathbf{n}^{ \pm}$is the outward unit normal vector to $\mathbf{K}^{ \pm}$. For a boundary face $\mathbf{F} \in \partial \mathbf{K}+\cap \partial \Omega$ these expressions are modified as

$$
\{\mathbf{v}\}_{F}=\mathbf{v}^{+} \text {, and } \llbracket \mathbf{v} \rrbracket_{F}=\mathbf{n}^{+} \times \mathbf{v}^{+} \text {. }
$$

Now we introduce the discontinuous finite element spaces and some basic operations on these spaces for later use. Let $\mathbb{P}_{p}(D)$ denote the space of polynomial functions of degree at most $\mathrm{p}$ in a domain $\mathrm{D}$. Let $\mathbf{V}_{\mathrm{h}}$ the global approximation space given by

$$
\mathbf{V}_{h}=\left\{\mathbf{v} \in\left[L^{2}(\Omega)\right]^{3} \mid \mathbf{v}_{\left.\right|_{K_{e}}} \in\left[\mathbb{P}_{p_{e}}\left(K_{e}\right)\right]^{3}, \quad \forall K_{e} \in \mathcal{T}_{h}\right\} .
$$

Note that this approximation space is common to DG and HDG methods. It represents the space where we seek an approximation of the electromagnetic field. We also introduce a traced finite element space $\mathbf{M}_{\mathrm{h}}$ defined as

$$
\mathbf{M}_{h}=\left\{\boldsymbol{\eta} \in\left[L^{2}\left(\mathcal{F}_{h}\right)\right]^{3}\left|\boldsymbol{\eta}_{\left.\right|_{F_{f}}} \in\left[\mathbb{P}_{p_{f}}\left(F_{f}\right)\right]^{3},(\boldsymbol{\eta} \cdot \mathbf{n})\right|_{F_{f}}=0, \quad \forall F_{f} \in \mathcal{F}_{h}\right\} .
$$

This space is specific to the HDG method, it represents the approximation space for traces (particularly for the hybrid variable that we will define in the following). For two vectorial functions $\mathbf{u}$ et $\mathbf{v}$ in $\left[L^{2}(D)\right]^{3}$, we denote $(\mathbf{u}, \mathbf{v})_{D}=\int_{D} \mathbf{u} \cdot \overline{\mathbf{v}} \mathrm{d} \mathbf{x}$ where $D$ is a domain of $\mathbb{R}^{3}$, and we denote $\langle\mathbf{u}, \mathbf{v}\rangle_{\mathrm{F}}=\int_{\mathrm{F}} \mathbf{u} \cdot \overline{\mathbf{v}}$ ds where $\mathrm{F}$ is a two-dimensional face. Accordingly, for the mesh $\mathcal{T}_{\mathrm{h}}$ we write

Finally we set

$$
\begin{aligned}
(\cdot, \cdot)_{\mathcal{T}_{\mathrm{h}}}=\sum_{\mathrm{K} \in \mathcal{T}_{\mathrm{h}}}(\cdot, \cdot)_{\mathrm{K}}, & \langle\cdot, \cdot\rangle_{\partial \mathcal{T}_{\mathrm{h}}}=\sum_{\mathrm{K} \in \mathcal{T}_{\mathrm{h}}}\langle\cdot, \cdot\rangle_{\partial \mathrm{K}}, \\
\langle\cdot, \cdot\rangle_{\mathcal{F}_{\mathrm{h}}}=\sum_{\mathrm{F} \in \mathcal{F}_{\mathrm{h}}}\langle\cdot, \cdot\rangle_{\mathrm{F}}, & \langle\cdot, \cdot\rangle_{\Gamma_{\mathrm{a}}}=\sum_{\mathrm{F} \in \mathcal{F}_{\mathrm{h}} \cap \Gamma_{\mathrm{a}}}\langle\cdot, \cdot\rangle_{\mathrm{F}} .
\end{aligned}
$$

$$
\gamma_{t}(\mathbf{v})=-\mathbf{n} \times(\mathbf{n} \times \mathbf{v}), \quad \gamma_{n}(\mathbf{v})=\mathbf{n}(\mathbf{n} \cdot \mathbf{v}),
$$

where $\gamma_{t}(\mathbf{v})$ and $\gamma_{n}(\mathbf{v})$ denote the tangential and normal components of $\mathbf{v}$ on a face of unit normal $\mathbf{n}$, and $\mathbf{v}=\gamma_{\mathbf{t}}(\mathbf{v})+\gamma_{\mathbf{n}}(\mathbf{v})$. 


\section{3 | PRINCIPLES AND GENERAL FORMULATION OF THE HDG METHOD}

As in a classical DG method we seek an approximation of the electromagnetic field solution of 1], denoted by $\left(\mathbf{E}_{\mathrm{h}}, \mathbf{H}_{\mathrm{h}}\right)$, in the space $\mathbf{V}_{\mathrm{h}} \times \mathrm{V}_{\mathrm{h}}$ such that for all $\mathrm{K}$ in $\mathcal{T}_{\mathrm{h}}$

$$
\left\{\begin{array}{l}
\left(\mathrm{i} \omega \varepsilon_{\mathrm{r}} \mathbf{E}_{\mathrm{h}}, \mathbf{v}\right)_{\mathrm{K}}-\left(\operatorname{curl} \mathbf{H}_{\mathrm{h}}, \mathbf{v}\right)_{\mathrm{K}}=0, \forall \mathbf{v} \in \mathbf{V}_{\mathrm{h}} \\
\left(\mathrm{i} \omega \mu_{\mathrm{r}} \mathbf{H}_{\mathrm{h}}, \mathbf{v}\right)_{\mathrm{K}}+\left(\operatorname{curl} \mathbf{E}_{\mathrm{h}}, \mathbf{v}\right)_{\mathrm{K}}=0, \forall \mathbf{v} \in \mathbf{V}_{\mathrm{h}}
\end{array}\right.
$$

Numerical traces $\widehat{\mathbf{E}}_{\mathrm{h}}$ and $\widehat{\mathbf{H}}_{\mathrm{h}}$ are introduced by applying appropriate Green's formulas

$$
\left\{\begin{array}{l}
\left(\mathrm{i} \omega \varepsilon_{\mathrm{r}} \mathbf{E}_{\mathrm{h}}, \mathbf{v}\right)_{\mathrm{K}}-\left(\mathbf{H}_{\mathrm{h}}, \mathbf{c u r l} \mathbf{v}\right)_{\mathrm{K}}+\left\langle\widehat{\mathbf{H}}_{\mathrm{h}}, \mathbf{n} \times \mathbf{v}\right\rangle_{\partial \mathrm{K}}=0, \forall \mathbf{v} \in \mathbf{V}_{\mathrm{h}}, \\
\left(\mathrm{i} \omega \mu_{\mathrm{r}} \mathbf{H}_{\mathrm{h}}, \mathbf{v}\right)_{\mathrm{K}}+\left(\mathbf{E}_{\mathrm{h}}, \mathbf{c u r l} \mathbf{v}\right)_{\mathrm{K}}-\left\langle\widehat{\mathbf{E}}_{\mathrm{h}}, \mathbf{n} \times \mathbf{v}\right\rangle_{\partial \mathrm{K}}=0, \forall \mathbf{v} \in \mathbf{V}_{\mathrm{h}} .
\end{array}\right.
$$

The key to define a DG method lies in the definition of the numerical traces. It enables to weakly enforce the continuity conditions between neighboring elements and thus ensures the consistency of the method. In a classical DG method we couple the local traces of the electromagnetic field $\mathbf{E}_{\mathrm{h}}$ and $\mathbf{H}_{\mathrm{h}}$ between the neighboring elements

$$
\widehat{\mathbf{E}}_{h}=\left\{\mathbf{E}_{h}\right\}+\alpha_{H} \llbracket \mathbf{H}_{h} \rrbracket \text { and } \widehat{\mathbf{H}}_{h}=\left\{\mathbf{H}_{h}\right\}+\alpha_{E} \llbracket \mathbf{E}_{h} \rrbracket,
$$

where $\alpha_{H}$ and $\alpha_{\mathrm{E}}$ are positive penalty parameters. To approximate the electromagnetic field $\left(\mathbf{E}_{\mathrm{h}}, \mathbf{H}_{\mathrm{h}}\right)$ for an element $\mathrm{K}$ of the mesh $\mathcal{T}_{\mathrm{h}}$ we need the value of the field of each neighboring element of $\mathrm{K}$, i.e., we need all degrees of freedom of the neighboring elements. Consequently a classical DG method leads to a global linear system to solve and the globally coupled degrees of freedom is

$$
\sum_{e=1}^{\left|\mathcal{T}_{h}\right|} 6 N_{K}^{e}
$$

where $\mathrm{N}_{\mathrm{K}}^{\mathrm{e}}$ is the dimension of the space $\mathbb{P}_{\mathrm{p}_{\mathrm{e}}}\left(\mathrm{K}_{\mathrm{e}}\right)$, i.e., $\mathrm{N}_{\mathrm{K}}^{\mathrm{e}}=\left(\mathrm{p}_{\mathrm{e}}+1\right)\left(\mathrm{p}_{\mathrm{e}}+2\right)\left(\mathrm{p}_{\mathrm{e}}+3\right) / 6$.

The aim of the HDG method is to reduce substantially the number of the globally coupled degrees of freedom. The key to define the HDG method lies in the definition of an hybrid variable which represents an additional unknown on on each face of $\mathcal{F}_{\mathrm{h}}$. A socalled conservativity condition is imposed on the numerical trace, whose definition involved the hybrid variable, at the interface between neighboring elements. As result, the HDG method leads to a linear system in terms of the degrees of freedom of the hybrid variable only. In this way, the number of globally coupled degrees of freedom is reduced. The local values of the electromagnetic fields can be obtained by solving local problems element-by-element. For the proposed HDG method (formulated in next section) the number of globally coupled degrees of freedom is

$$
\sum_{f=1}^{\left|\mathcal{F}_{h}\right|} 2 N_{F}^{f}
$$

where $N_{F}^{f}$ is the dimension of the space $\mathbb{P}_{p_{f}}\left(F_{f}\right)$, i.e., $N_{F}^{f}=\left(p_{f}+1\right)\left(p_{f}+2\right) / 2$.

Remark 1. Assuming the interpolation degrees are $\mathrm{p}_{\mathrm{e}}=\mathrm{p}_{\mathrm{f}}=\mathrm{p}, \forall \mathrm{K}_{\mathrm{e}} \in \mathcal{T}_{\mathrm{h}}, \forall \mathrm{F}_{\mathrm{f}} \in \mathcal{F}_{\mathrm{h}}$, the number of globally coupled degrees of freedom is then

$$
\begin{array}{ll}
\text { DGM: } & (p+1)(p+2)(p+3)\left|\mathcal{T}_{h}\right|, \\
\text { HDGM: } & (p+1)(p+2)\left|\mathcal{F}_{h}\right| .
\end{array}
$$

For a simplicial mesh $\left|\mathcal{F}_{h}\right| \approx 2\left|\mathcal{T}_{h}\right|$, the ratio of the globally coupled degrees of freedom is roughly $2 /(\mathrm{p}+3)$ for HDG method over DG method.

Now we are interested in the formulation of the HDG method. First note that $\mathbf{n} \times \mathbf{v}=\mathbf{n} \times \gamma_{\mathrm{t}}(\mathbf{v})$, thus the system 6 is equivalent to

$$
\left\{\begin{array}{l}
\left(\mathrm{i} \omega \varepsilon_{\mathrm{r}} \mathbf{E}_{\mathrm{h}}, \mathbf{v}\right)_{\mathrm{K}}-\left(\mathbf{H}_{\mathrm{h}}, \text { curl v }\right)_{\mathrm{K}}+\left\langle\gamma_{\mathrm{t}}\left(\widehat{\mathbf{H}}_{\mathrm{h}}\right), \mathbf{n} \times \mathbf{v}\right\rangle_{\partial \mathrm{K}}=0, \forall \mathbf{v} \in \mathbf{V}_{\mathrm{h}}, \\
\left(\mathrm{i} \omega \mu_{\mathrm{r}} \mathbf{H}_{\mathrm{h}}, \mathbf{v}\right)_{\mathrm{K}}+\left(\mathbf{E}_{\mathrm{h}}, \mathbf{c u r l} \mathbf{v}\right)_{\mathrm{K}}-\left\langle\gamma_{\mathrm{t}}\left(\widehat{\mathbf{E}}_{\mathrm{h}}\right), \mathbf{n} \times \mathbf{v}\right\rangle_{\partial \mathrm{K}}=0, \forall \mathbf{v} \in \mathbf{V}_{\mathrm{h}} .
\end{array}\right.
$$

We introduce the hybrid variable $\boldsymbol{\Lambda}_{\mathrm{h}}$ defined by

$$
\boldsymbol{\Lambda}_{h}:=\gamma_{t}\left(\widehat{\mathbf{H}}_{h}\right), \quad \forall F \in \mathcal{F}_{h}
$$


In the HDG method we want to formulate the local fields in K through 8] assuming that $\boldsymbol{\Lambda}_{\mathrm{h}}$ is known on all faces of an element $\mathrm{K}$. In order to achieve this, we consider a numerical trace $\gamma_{t}\left(\widehat{\mathbf{E}}_{\mathrm{h}}\right)$ of the form

$$
\gamma_{t}\left(\widehat{\mathbf{E}}_{h}\right)=\gamma_{t}\left(\mathbf{E}_{h}\right)+\tau^{K} \mathbf{n} \times\left(\boldsymbol{\Lambda}_{h}-\gamma_{t}\left(\mathbf{H}_{h}\right)\right) \text { on } \partial K
$$

where $\tau^{\mathrm{K}}$ is a local stabilization parameter. We note that the definition of the numerical trace $\Lambda_{\mathrm{h}}$ is directly linked to one of the jump conditions that apply to the electric and magnetic field at a material interface. Indeed, one of these conditions states that the tangential component of the magnetic field is continuous, which is exactly what will be obtained from the definition of the hybrid variable. Besides, the numerical trace $\gamma_{t}\left(\widehat{\mathbf{E}}_{h}\right)$ is defined in terms of the tangential components of the local electric and magnetic fields in $\mathrm{K}$ so that to ensure that the tangential component of the magnetic field matches the continuous value given by the trace $\Lambda_{\mathrm{h}}$ at convergence.

Remark 2. Once the hybrid variable $\boldsymbol{\Lambda}_{\mathrm{h}}$ is obtained on all the faces of an element $\mathrm{K}$ the electromagnetic field inside this element can be solved through the associated local linear system 8 using the numerical traces defined by 9 and 10 .

Remark 3. It can be shown 9 that the numerical traces 9 and 10 can be expressed in a form similar to the one of the definition of the classical DG numerical traces [7. In particular, if we take $\tau$ uniformly equal to 1, the HDG numerical traces coincide with the upwind flux-based DG formulation. In oher words, the HDG numerical traces implicitly introduce artificial dissipation in the scheme therefore preventing the appearance of spurious solutions albeit the fully discrete scheme makes use of scalar polynomials to expand the electric and magnetic fields as will be seen in the sequel.

Adding all contributions of 8 over all elements and enforcing the continuity of the tangential component of $\widehat{\mathbf{E}}_{\mathrm{h}}$, we can formulate the following problem:

find $\left(\mathbf{E}_{\mathrm{h}}, \mathbf{H}_{\mathrm{h}}, \boldsymbol{\Lambda}_{\mathrm{h}}\right) \in \mathbf{V}_{\mathrm{h}} \times \mathbf{V}_{\mathrm{h}} \times \mathbf{M}_{\mathrm{h}}$ such that

$$
\left\{\begin{aligned}
\left(\mathrm{i} \omega \varepsilon_{\mathrm{r}} \mathbf{E}_{\mathrm{h}}, \mathbf{v}\right)_{\mathcal{T}_{\mathrm{h}}} & -\left(\mathbf{H}_{\mathrm{h}}, \mathbf{c u r l} \mathbf{v}\right)_{\mathcal{T}_{\mathrm{h}}} \\
& +\left\langle\boldsymbol{\Lambda}_{\mathrm{h}}, \mathbf{n} \times \mathbf{v}\right\rangle_{\partial \mathcal{T}_{\mathrm{h}}}=0, \forall \mathbf{v} \in \mathbf{V}_{\mathrm{h}}, \\
\left(\mathrm{i} \omega \mu_{\mathrm{r}} \mathbf{H}_{\mathrm{h}}, \mathbf{v}\right)_{\mathcal{T}_{\mathrm{h}}} & +\left(\mathbf{E}_{\mathrm{h}}, \mathbf{c u r l} \mathbf{v}\right)_{\mathcal{T}_{\mathrm{h}}} \\
& -\left\langle\gamma_{\mathrm{t}}\left(\widehat{\mathbf{E}}_{\mathrm{h}}\right), \mathbf{n} \times \mathbf{v}\right\rangle_{\partial \mathcal{T}_{\mathrm{h}}}=0, \forall \mathbf{v} \in \mathbf{V}_{\mathrm{h}}, \\
\left\langle\llbracket \gamma_{\mathrm{t}}\left(\widehat{\mathbf{E}}_{\mathrm{h}}\right) \rrbracket, \boldsymbol{\eta}\right\rangle_{\mathcal{F}_{\mathrm{h}}} & -\left\langle\boldsymbol{\Lambda}_{\mathrm{h}}, \boldsymbol{\eta}\right\rangle_{\Gamma_{\mathrm{a}}}=\left\langle\mathbf{g}^{\text {inc }}, \boldsymbol{\eta}\right\rangle_{\Gamma_{\mathrm{a}}}, \forall \boldsymbol{\eta} \in \mathbf{M}_{\mathrm{h}},
\end{aligned}\right.
$$

where the last equation is called the conservativity condition, with which we ask the tangential component of $\widehat{\mathbf{E}}_{\mathrm{h}}$ to be weakly continuous across any interfaces between neighboring elements. With the definition of $\gamma_{t}\left(\widehat{\mathbf{E}}_{h}\right)$ we employ again a Green formula in the second equation of 11 to obtain the following problem: find $\left(\mathbf{E}_{\mathrm{h}}, \mathbf{H}_{\mathrm{h}}, \boldsymbol{\Lambda}_{\mathrm{h}}\right) \in \mathbf{V}_{\mathrm{h}} \times \mathbf{V}_{\mathrm{h}} \times \mathbf{M}_{\mathrm{h}}$ such that

Note that we have used

$$
\left\{\begin{aligned}
\left(\mathrm{i} \omega \varepsilon_{\mathrm{r}} \mathbf{E}_{\mathrm{h}}, \mathbf{v}\right)_{\mathcal{T}_{\mathrm{h}}} & -\left(\mathbf{H}_{\mathrm{h}}, \mathbf{c u r l} \mathbf{v}\right)_{\mathcal{T}_{\mathrm{h}}} \\
& +\left\langle\boldsymbol{\Lambda}_{\mathrm{h}}, \mathbf{n} \times \mathbf{v}\right\rangle_{\partial \mathcal{T}_{\mathrm{h}}}=0, \forall \mathbf{v} \in \mathbf{V}_{\mathrm{h}}, \\
\left(\mathrm{i} \omega \mu_{\mathrm{r}} \mathbf{H}_{\mathrm{h}}, \mathbf{v}\right)_{\mathcal{T}_{\mathrm{h}}} & +\left(\operatorname{curl} \mathbf{E}_{\mathrm{h}}, \mathbf{v}\right)_{\mathcal{T}_{\mathrm{h}}} \\
& +\left\langle\tau \mathbf{n} \times\left(\mathbf{H}_{\mathrm{h}}-\boldsymbol{\Lambda}_{\mathrm{h}}\right), \mathbf{n} \times \mathbf{v}\right\rangle_{\partial \mathcal{T}_{\mathrm{h}}}=0, \forall \mathbf{v} \in \mathbf{V}_{\mathrm{h}}, \\
\left\langle\mathbf{n} \times \mathbf{E}_{\mathrm{h}}, \boldsymbol{\eta}\right\rangle_{\partial \mathcal{T}_{\mathrm{h}}} & +\left\langle\tau\left(\gamma_{\mathrm{t}}\left(\mathbf{H}_{\mathrm{h}}\right)-\boldsymbol{\Lambda}_{\mathrm{h}}\right), \boldsymbol{\eta}\right\rangle_{\partial \mathcal{T}_{\mathrm{h}}} \\
& -\left\langle\boldsymbol{\Lambda}_{\mathrm{h}}, \boldsymbol{\eta}\right\rangle_{\Gamma_{\mathrm{a}}}=\left\langle\mathbf{g}^{\mathrm{inc}}, \boldsymbol{\eta}\right\rangle_{\Gamma_{\mathrm{a}}}, \forall \boldsymbol{\eta} \in \mathbf{M}_{\mathrm{h}} .
\end{aligned}\right.
$$

$$
\mathbf{n} \times \gamma_{t}(\mathbf{v})=\mathbf{n} \times \mathbf{v} \text { and } \mathbf{n} \times\left(\mathbf{n} \times \gamma_{t}(\mathbf{v})\right)=-\gamma_{t}(\mathbf{v})
$$

to obtain 12.

In summary we can decomposed the HDG method in two steps: 
1. A conservativity condition (third equation of [12]) is imposed on the numerical trace, whose definition involved the hybrid variable at the interface between neighboring elements. As result we obtain a global linear system in terms of the degrees of freedom of the hybrid variable;

2. Once the degrees of freedom of the hybrid variable are known, the local values of the electromagnetic fields can be obtained by solving local linear systems element-by-element from the first and the second equation of 12 .

\section{4 | IMPLEMENTATION OF THE HDG METHOD}

In this section we outline the main steps in the formulation of the discrete HDG method. In short, there ar two main ingredients: (1) matrix formulations of local solvers to approximate the local values (i.e., the degrees of freedom) of the electromagnetic fields element-by-element; (2) a matrix formulation of the global solver to approximate the values of the numerical trace, i.e., the degrees of freedom of the hybrid variable, on each face of $\mathcal{F}_{\mathrm{h}}$.

\section{1 | Preliminaries}

First we introduce some notations and definitions for later use. We denote the restriction of the electromagnetic field on an element $\mathrm{K}_{\mathrm{e}}$ in $\mathcal{T}_{\mathrm{h}}$ by $\left(\left.\mathbf{E}_{\mathrm{h}}\right|_{\mathrm{K}_{\mathrm{e}}},\left.\mathbf{H}_{\mathrm{h}}\right|_{\mathrm{K}_{\mathrm{e}}}\right)=\left(\mathbf{E}^{\mathrm{e}}, \mathbf{H}^{\mathrm{e}}\right): \mathrm{K}_{\mathrm{e}} \times \mathrm{K}_{\mathrm{e}} \longrightarrow \mathbb{C}^{3} \times \mathbb{C}^{3}$, where $\mathbf{E}^{\mathrm{e}}(\mathbf{x})=\left[\mathrm{E}_{\mathrm{x}}^{\mathrm{e}}(\mathbf{x}), \mathrm{E}_{\mathrm{y}}^{\mathrm{e}}(\mathbf{x}), \mathrm{E}_{\mathrm{z}}^{\mathrm{e}}(\mathbf{x})\right]^{\top}$ and $\mathbf{H}^{\mathrm{e}}(\mathbf{x})=\left[\mathrm{H}_{\mathrm{x}}^{\mathrm{e}}(\mathbf{x}), \mathrm{H}_{\mathrm{y}}^{\mathrm{e}}(\mathbf{x}), \mathrm{H}_{\mathrm{z}}^{\mathrm{e}}(\mathbf{x})\right]^{\top}$. As in a classical DG method for each element $\mathrm{K}_{\mathrm{e}}$ we seek an approximation of the components of the electromagnetic fields by a linear combination of basis functions $\varphi_{j}^{e}(\mathbf{x})$ of the space $\mathbb{P}_{\mathrm{p}_{\mathrm{e}}}\left(\mathrm{K}_{\mathrm{e}}\right)$, i.e.,

$$
E_{\xi}^{e}(\mathbf{x})=\sum_{j=1}^{N_{K}^{e}} \underline{E}_{\xi}^{e}[j] \varphi_{j}^{e}(\mathbf{x}), \quad H_{\xi}^{e}(\mathbf{x})=\sum_{j=1}^{N_{K}^{e}} \underline{H}_{\xi}^{e}[j] \varphi_{j}^{e}(\mathbf{x}) \quad(\xi \in\{x, y, z\}),
$$

where $\underline{E}_{\xi}^{e}[j], \underline{H}_{\xi}^{e}[j]$ represent the degrees of freedom of the electromagnetic field in $\mathrm{K}^{\mathrm{e}}$ and $\mathrm{N}_{\mathrm{K}}^{\mathrm{e}}$ is the dimension of the space $\mathbb{P}_{\mathrm{p}_{\mathrm{e}}}\left(\mathrm{K}_{\mathrm{e}}\right)$. Similarly for a face $F_{f}$ in $\mathcal{F}_{h}$ we denote $\left.\boldsymbol{\Lambda}_{h}\right|_{F_{f}}=\Lambda^{f}: F_{f} \longrightarrow \mathbb{C}^{3}$ and we set

$$
\mathbf{\Lambda}^{f}(\mathbf{x})=\Lambda_{\mathbf{u}}^{f}(\mathbf{x}) \mathbf{u}^{f}+\Lambda_{\mathbf{w}}^{f}(\mathbf{x}) \mathbf{w}^{f},
$$

where $\mathbf{u}^{f}$ and $\mathbf{w}^{f}$ are coordinate axis (not necessarily orthogonal). We seek an approximation of $\Lambda_{\mathbf{u}}^{f}$ and $\Lambda_{\mathbf{w}}^{f}$ by a linear combination of basis functions $\psi_{\mathrm{j}}^{\mathrm{f}}(\mathbf{x})$ of the space $\mathbb{P}_{\mathrm{p}_{\mathrm{f}}}\left(\mathrm{F}_{\mathrm{f}}\right)$, i.e.,

$$
\Lambda_{\mathbf{u}}^{f}(\mathbf{x})=\sum_{j=1}^{N_{F}^{f}} \underline{\Lambda}_{\mathbf{u}}^{f}[j] \psi_{j}^{f}(\mathbf{x}), \quad \Lambda_{\mathbf{w}}^{f}(\mathbf{x})=\sum_{j=1}^{N_{F}^{f}} \underline{\Lambda}_{\mathbf{w}}^{f}[j] \psi_{j}^{f}(\mathbf{x}),
$$

where $\underline{\Lambda}_{\mathrm{u}}^{\mathrm{f}}[\mathrm{j}], \underline{\Lambda}_{\mathrm{w}}^{\mathrm{f}}[\mathrm{j}]$ are the degrees of freedom of the components of $\Lambda^{\mathrm{f}}$ associated with the face $\mathrm{F}^{\mathrm{f}}$, and $\mathrm{N}_{\mathrm{F}}^{\mathrm{f}}$ is the dimension of the space $\mathbb{P}_{\mathrm{p}_{\mathrm{f}}}\left(\mathrm{F}_{\mathrm{f}}\right)$.

Remark 4. In practice, we set $\mathbf{u}^{f}=\overline{\mathbf{u}}^{\mathrm{f}} /\left\|\overline{\mathbf{u}}^{\mathrm{f}}\right\|_{2}$ and $\mathbf{w}^{\mathrm{f}}=\overline{\mathbf{w}}^{\mathrm{f}} /\left\|\overline{\mathbf{w}}^{\mathrm{f}}\right\|_{2}$ with $\overline{\mathbf{u}}^{\mathrm{f}}=\mathrm{n}_{2}^{\mathrm{f}}-\mathrm{n}_{1}^{\mathrm{f}}$ and $\overline{\mathbf{w}}^{\mathrm{f}}=\mathrm{n}_{3}^{\mathrm{f}}-\mathrm{n}_{1}^{\mathrm{f}}$, where $\mathrm{n}_{1}^{\mathrm{f}}$, $\mathrm{n}_{2}^{\mathrm{f}}$ and $\mathrm{n}_{3}^{\mathrm{f}}$ are the three nodes of the face $F_{f}$. Since the outward normal $\mathbf{n}$ can be computed through $\mathbf{u}^{\mathrm{f}} \times \mathbf{w}^{\mathrm{f}}$ we can easily show that $\left.\left(\mathbf{n} \cdot \boldsymbol{\Lambda}_{\mathrm{h}}^{\mathrm{f}}\right)\right|_{\mathrm{F}_{\mathrm{f}}}=0$ in accordance with the definition of the space $\mathrm{M}_{\mathrm{h}}$ given by 4 .

We denote by $\nu_{\mathrm{e}}$ the set of indices of the elements which are neighbors of $\mathrm{K}_{\mathrm{e}}$ (having an interface in common). Thus for each element of the mesh $\mathrm{K}_{\mathrm{e}_{\mathrm{i}}} \in \mathcal{T}_{\mathrm{h}}\left(\mathrm{i} \in\left\{1, \cdots,\left|\mathcal{T}_{\mathrm{h}}\right|\right)\right.$ we associate $\left|\nu_{\mathrm{e}_{\mathrm{i}}}\right|$ faces, denoted $\partial \mathrm{K}_{\mathrm{e}_{\mathrm{i}}} \in \partial \mathcal{T}_{\mathrm{h}}$, defined by

$$
\partial K_{e_{i}}^{l}=\overline{K_{e_{i}}} \cap \overline{K_{e_{j}}}, \quad l \in\left\{1, \cdots,\left|\nu_{e_{i}}\right|\right\}, \quad j \in \nu_{e_{i}} .
$$

As it will be useful later, let us define an index mapping function (local to global), denoted by $\sigma$, graphically depicted in Fig. 1 and defined as follow

$$
\left\{\begin{array}{l}
\forall \mathrm{F}_{\mathrm{f}} \in \mathcal{F}_{\mathrm{h}}^{\mathrm{l}} \text { such that } \mathrm{F}_{\mathrm{f}}=\partial \mathrm{K}_{\mathrm{e}}^{\mathrm{l}} \cap \partial \mathrm{K}_{\mathrm{g}}^{\mathrm{k}}, \quad \sigma(\mathrm{e}, \mathrm{l})=\sigma(\mathrm{g}, \mathrm{k})=\mathrm{f}, \\
\forall \mathrm{F}_{\mathrm{f}} \in \mathcal{F}_{\mathrm{h}}^{\mathrm{B}} \text { such that } \mathrm{F}_{\mathrm{f}}=\partial \mathrm{K}_{\mathrm{e}}^{\mathrm{I}} \cap\left(\Gamma_{\mathrm{m}} \cup \Gamma_{\mathrm{m}}\right), \quad \sigma(\mathrm{e}, \mathrm{l})=\mathrm{f}
\end{array}\right.
$$



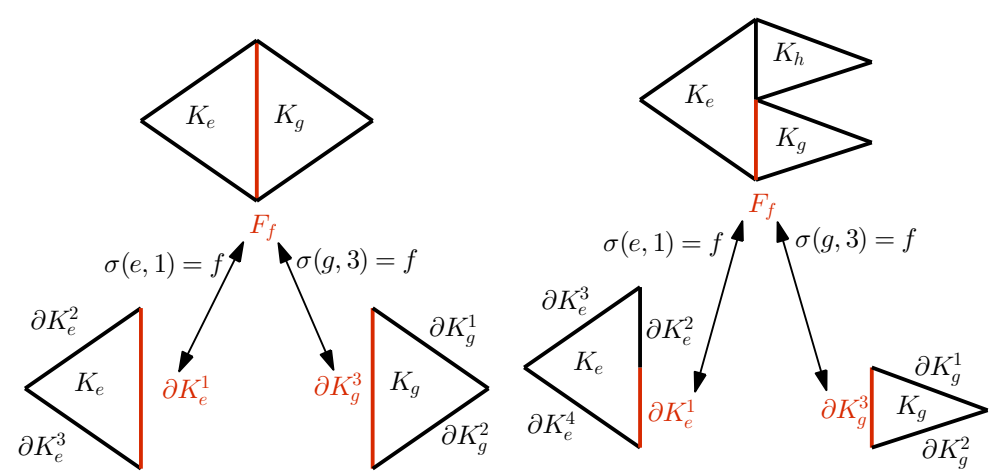

FIGURE 1 Diagram (2D) demonstrating the use of the index mapping function (local to global) $\sigma$ described in the text (conforming case on left / non-conforming case on right).

\subsection{Discretization of local problems}

To get the discretization of the first and second equation of 12 we first note that for all $\mathbf{v} \in \mathbf{V}_{\mathrm{h}}$ we have

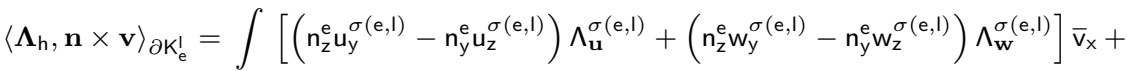

$$
\begin{aligned}
& \partial \mathrm{K}_{\mathrm{e}}^{\mathrm{l}}\left[\left(\mathrm{n}_{\mathrm{x}}^{\mathrm{e}} \mathrm{u}_{\mathrm{z}}^{\sigma(\mathrm{e}, \mathrm{l})}-\mathrm{n}_{\mathrm{z}}^{\mathrm{e}} \mathrm{u}_{\mathrm{x}}^{\sigma(\mathrm{e}, \mathrm{l})}\right) \Lambda_{\mathrm{u}}^{\sigma(\mathrm{e}, \mathrm{l})}+\left(\mathrm{n}_{\mathrm{x}}^{\mathrm{e}} \mathrm{w}_{\mathrm{z}}^{\sigma(\mathrm{e}, \mathrm{l})}-\mathrm{n}_{\mathrm{z}}^{\mathrm{e}} \mathrm{w}_{\mathrm{x}}^{\sigma(\mathrm{e}, \mathrm{l})}\right) \Lambda_{\mathrm{w}}^{\sigma(\mathrm{e}, \mathrm{l})}\right] \overline{\mathrm{v}}_{\mathrm{y}}+ \\
& {\left[\left(n_{y}^{e} u_{x}^{\sigma(e, l)}-n_{x}^{e} u_{y}^{\sigma(e, l)}\right) \Lambda_{u}^{\sigma(e, l)}+\left(n_{y}^{e} w_{x}^{\sigma(e, l)}-n_{x}^{e} w_{y}^{\sigma(e, l)}\right) \Lambda_{w}^{\sigma(e, l)}\right] \bar{v}_{z} d s,} \\
& \left\langle\mathbf{n} \times \boldsymbol{\Lambda}_{\mathrm{h}}, \mathbf{n} \times \mathbf{v}\right\rangle_{\partial \mathrm{K}_{\mathrm{e}}^{1}}=\left\langle-\mathbf{n} \times\left(\mathbf{n} \times \boldsymbol{\Lambda}_{\mathrm{h}}\right), \mathbf{v}\right\rangle_{\partial \mathrm{K}_{\mathrm{e}}^{1}}=\left\langle\boldsymbol{\Lambda}_{\mathrm{h}}, \mathbf{v}\right\rangle_{\partial \mathrm{K}_{\mathrm{e}}^{1}}= \\
& \int_{\partial \mathrm{K}_{\mathrm{e}}^{\mathrm{I}}}\left(\mathrm{u}_{\mathrm{x}}^{\sigma(\mathrm{e}, \mathrm{l})} \Lambda_{\mathbf{u}}^{\sigma(\mathrm{e}, \mathrm{l})}+\mathrm{w}_{\mathrm{x}}^{\sigma(\mathrm{e}, \mathrm{l})} \Lambda_{\mathrm{w}}^{\sigma(\mathrm{e}, \mathrm{l})}\right) \overline{\mathrm{v}}_{\mathrm{x}}+\left(\mathrm{u}_{\mathrm{y}}^{\sigma(\mathrm{e}, \mathrm{l})} \Lambda_{\mathbf{u}}^{\sigma(\mathrm{e}, \mathrm{l})}+\mathrm{w}_{\mathrm{y}}^{\sigma(\mathrm{e}, \mathrm{l})} \Lambda_{\mathrm{w}}^{\sigma(\mathrm{e}, \mathrm{l})}\right) \overline{\mathrm{v}}_{\mathrm{y}}+\left(\mathrm{u}_{\mathrm{z}}^{\sigma(\mathrm{e}, \mathrm{l})} \Lambda_{\mathbf{u}}^{\sigma(\mathrm{e}, \mathrm{l})}+\mathrm{w}_{\mathrm{z}}^{\sigma(\mathrm{e}, \mathrm{l})} \Lambda_{\mathrm{w}}^{\sigma(\mathrm{e}, \mathrm{l})}\right) \overline{\mathrm{v}}_{\mathrm{z}} \mathrm{ds} .
\end{aligned}
$$

Then, using 13 - 14 and the basis functions of the space $V_{h}$ as test functions in the first and the second equation of the system 12, we can formulate the local linear system associated with the element $\mathrm{K}_{\mathrm{e}}$ as

$$
\mathbb{A}^{e}\left[\begin{array}{l}
\underline{\mathrm{E}}_{\mathrm{x}}^{\mathrm{e}} \\
\underline{\mathrm{E}}_{\mathrm{y}}^{\mathrm{e}} \\
\underline{\mathrm{E}}_{\mathrm{z}}^{\mathrm{e}} \\
\underline{\mathrm{H}}_{\mathrm{y}}^{\mathrm{e}} \\
\underline{\mathrm{H}}_{\mathrm{x}}^{\mathrm{e}} \\
\underline{\mathrm{H}}_{\mathrm{z}}^{\mathrm{e}}
\end{array}\right]+\sum_{l=1}^{\left|\nu_{e}\right|} \mathbb{C}^{(e, l)}\left[\begin{array}{l}
\underline{\Lambda}_{\mathbf{u}}^{\sigma(\mathrm{e}, \mathrm{l})} \\
\underline{\Lambda}_{\mathrm{w}}^{\sigma(\mathrm{e}, \mathrm{l})}
\end{array}\right]=0,
$$

where $\mathbb{A}^{\mathrm{e}}$ matrix of size $6 \mathrm{~N}_{\mathrm{K}}^{\mathrm{e}} \times 6 \mathrm{~N}_{\mathrm{K}}^{\mathrm{e}}$, while $\underline{E}_{\xi}^{\mathrm{e}}, \underline{H}_{\xi}^{\mathrm{e}}$ and $\underline{\Lambda}_{\zeta}^{\sigma(\mathrm{e}, \mathrm{l})}$ are the column vectors of degrees of freedom

$$
\begin{aligned}
\underline{\mathrm{E}}_{\xi}^{\mathrm{e}} & =\left[\underline{\mathrm{E}}_{\xi}^{\mathrm{e}}[1], \cdots, \underline{\mathrm{E}}_{\xi}^{\mathrm{e}}\left[\mathrm{N}_{\mathrm{K}}^{\mathrm{e}}\right]\right]^{\top}, \quad \xi \in\{\mathrm{x}, \mathrm{y}, \mathrm{z}\}, \\
\underline{\mathrm{H}}_{\xi}^{\mathrm{e}} & =\left[\underline{\mathrm{H}}_{\xi}^{\mathrm{e}}[1], \cdots, \underline{\mathrm{H}}_{\xi}^{\mathrm{e}}\left[\mathrm{N}_{\mathrm{K}}^{\mathrm{e}}\right]\right]^{\top}, \quad \xi \in\{\mathrm{x}, \mathrm{y}, \mathrm{z}\}, \\
\underline{\Lambda}_{\nu}^{\sigma(\mathrm{e}, \mathrm{l})} & =\left[\underline{\Lambda}_{\nu}^{\sigma(\mathrm{e}, \mathrm{l})}[1], \cdots, \underline{\Lambda}_{\nu}^{\sigma(\mathrm{e}, \mathrm{l})}\left[\mathrm{N}_{\mathrm{F}}^{\sigma(\mathrm{e}, \mathrm{l})}\right]\right]^{\top}, \quad \boldsymbol{\nu} \in\{\mathbf{u}, \mathbf{w}\} .
\end{aligned}
$$




\subsection{Global discretization for the hybrid variable}

In this section we discretize the third equation of 12, i.e., the conservativity condition, to obtain a global linear system in terms of the degrees of freedom of the hybrid variable $\Lambda_{h}$. Let $\mathrm{F}_{\mathrm{f}} \in \mathcal{F}_{\mathrm{h}}^{1}$ an internal face shared by the elements $\mathrm{K}_{\mathrm{e}}$ and $\mathrm{K}_{\mathrm{g}}$ with local indices I and $\mathrm{k}$, respectively, i.e., $\mathrm{f}=\sigma(\mathrm{e}, \mathrm{l})=\sigma(\mathrm{g}, \mathrm{k})$. The conservativity condition for $\mathrm{F}_{\mathrm{f}}$ and for all $\boldsymbol{\eta} \in \mathbf{M}_{\mathrm{h}}$ can be written as

$$
\begin{aligned}
& \left\langle\mathbf{n} \times \mathbf{E}_{\mathrm{h}}, \boldsymbol{\eta}\right\rangle_{\partial \mathrm{K}_{\mathrm{e}}^{\mathrm{l}}}-\tau^{(\mathrm{e}, \mathrm{l})}\left\langle\mathbf{n} \times\left(\mathbf{n} \times \mathbf{H}_{\mathrm{h}}\right), \boldsymbol{\eta}\right\rangle_{\partial \mathrm{K}_{\mathrm{e}}^{\mathrm{l}}}-\tau^{(\mathrm{e}, \mathrm{l})}\left\langle\boldsymbol{\Lambda}_{\mathrm{h}}, \boldsymbol{\eta}\right\rangle_{\partial \mathrm{K}_{\mathrm{e}}^{\mathrm{I}}}+ \\
& \left\langle\mathbf{n} \times \mathbf{E}_{\mathrm{h}}, \boldsymbol{\eta}\right\rangle_{\partial \mathrm{K}_{\mathrm{g}}^{\mathrm{k}}}-\tau^{(\mathrm{g}, \mathrm{k})}\left\langle\mathbf{n} \times\left(\mathbf{n} \times \mathbf{H}_{\mathrm{h}}\right), \boldsymbol{\eta}\right\rangle_{\partial \mathrm{K}_{\mathrm{g}}^{\mathrm{k}}}-\tau^{(\mathrm{g}, \mathrm{k})}\left\langle\boldsymbol{\Lambda}_{\mathrm{h}}, \boldsymbol{\eta}\right\rangle_{\partial \mathrm{K}_{\mathrm{g}}^{\mathrm{k}}}=0 .
\end{aligned}
$$

For a boundary face $F_{f} \in \Gamma_{a}$ such that $F_{f} \in \partial K_{e}^{1} \cap \Gamma_{a}$, the conservativity condition for all $\boldsymbol{\eta} \in \mathbf{M}_{h}$ is given by

$$
\left\langle\mathbf{n} \times \mathbf{E}_{h}, \boldsymbol{\eta}\right\rangle_{\partial K_{e}^{l}}-\tau^{(e, l)}\left\langle\mathbf{n} \times\left(\mathbf{n} \times \mathbf{H}_{h}\right), \boldsymbol{\eta}\right\rangle_{\partial K_{e}^{l}}-\left(1+\tau^{(e, l)}\right)\left\langle\boldsymbol{\Lambda}_{h}, \boldsymbol{\eta}\right\rangle_{\partial K_{e}^{l}}=\left\langle\mathbf{g}^{i n c}, \boldsymbol{\eta}\right\rangle_{\partial K_{e}^{l}} .
$$

As it will be useful for the discretization of these latter equations, let us do some preliminary computations. For all $\boldsymbol{\eta} \in \mathbf{M}_{\mathrm{h}}$, we write

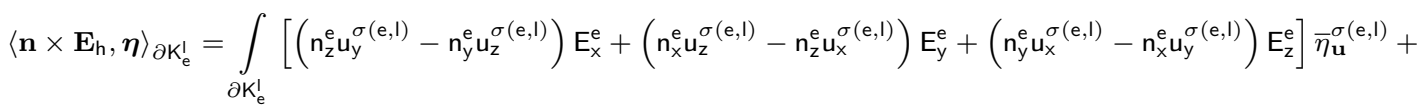

$$
\begin{aligned}
& {\left[\left(n_{z}^{e} w_{y}^{\sigma(e, l)}-n_{y}^{e} w_{z}^{\sigma(e, l)}\right) E_{x}^{e}+\left(n_{x}^{e} w_{z}^{\sigma(e, l)}-n_{z}^{e} w_{x}^{\sigma(e, l)}\right) E_{y}^{e}+\left(n_{y}^{e} w_{x}^{\sigma(e, l)}-n_{x}^{e} w_{y}^{\sigma(e, l)}\right) E_{z}^{e}\right] \bar{\eta}_{w}^{\sigma(e, l)} d s,} \\
& \left\langle-\mathbf{n} \times\left(\mathbf{n} \times \mathbf{H}_{\mathrm{h}}\right), \boldsymbol{\eta}\right\rangle_{\partial \mathrm{K}_{\mathrm{e}}}=\int_{\partial \mathrm{K}_{\mathrm{e}}^{\prime}}\left(\mathrm{u}_{\mathrm{x}}^{\sigma(\mathrm{e}, \mathrm{l})} \mathrm{H}_{\mathrm{x}}^{\mathrm{e}}+\mathrm{u}_{\mathrm{y}}^{\sigma(\mathrm{e}, \mathrm{l})} \mathrm{H}_{\mathrm{y}}^{\mathrm{e}}+\mathrm{u}_{\mathrm{z}}^{\sigma(\mathrm{e}, \mathrm{l})} \mathrm{H}_{\mathrm{z}}^{\mathrm{e}}\right) \bar{\eta}_{\mathbf{u}}^{\sigma(\mathrm{e}, \mathrm{l})}+\left(\mathrm{w}_{\mathrm{x}}^{\sigma(\mathrm{e}, \mathrm{l})} \mathrm{H}_{\mathrm{x}}^{\mathrm{e}}+\mathrm{w}_{\mathrm{y}}^{\sigma(\mathrm{e}, \mathrm{l})} \mathrm{H}_{\mathrm{y}}^{\mathrm{e}}+\mathrm{w} \sigma(\mathrm{e}, \mathrm{I})_{\mathrm{z}} \mathrm{H}_{\mathrm{z}}^{\mathrm{e}}\right) \bar{\eta}_{\mathbf{w}}^{\sigma(\mathrm{e}, \mathrm{ll})} \mathrm{ds}, \\
& \left\langle\boldsymbol{\Lambda}_{\mathrm{h}}, \boldsymbol{\eta}\right\rangle_{\partial \mathrm{K}_{\mathrm{e}}}=\int_{\partial \mathrm{K}_{\mathrm{e}}}\left[\Lambda_{\mathbf{u}}^{\sigma(\mathrm{e}, \mathrm{l})}+\left(\mathbf{u}^{\sigma(\mathrm{e}, \mathrm{l})} \cdot \mathbf{w}^{\sigma(\mathrm{e}, \mathrm{l})}\right) \Lambda_{\mathbf{w}}^{\sigma(\mathrm{e}, \mathrm{l})}\right] \bar{\eta}_{\mathbf{u}}^{\sigma(\mathrm{e}, \mathrm{l})}+\left[\Lambda_{\mathbf{w}}^{\sigma(\mathrm{e}, \mathrm{l})}+\left(\mathbf{u}^{\sigma(\mathrm{e}, \mathrm{l})} \cdot \mathbf{w}^{\sigma(\mathrm{e}, \mathrm{l})}\right) \Lambda_{\mathbf{u}}^{\sigma(\mathrm{e}, \mathrm{l})}\right] \bar{\eta}_{\mathbf{w}}^{\sigma(\mathrm{e}, \mathrm{l})} \mathrm{d} \mathbf{s} .
\end{aligned}
$$

Note that we have used the following equality for the second equation of 19 , $\forall \mathrm{F} \in \mathcal{F}_{\mathrm{h}}$

$$
\left\langle-\mathbf{n} \times\left(\mathbf{n} \times \mathbf{H}_{h}\right), \boldsymbol{\eta}\right\rangle_{F}=\left\langle\mathbf{n} \times \mathbf{H}_{h}, \mathbf{n} \times \boldsymbol{\eta}\right\rangle_{F}=\left\langle\mathbf{H}_{h},-\mathbf{n} \times(\mathbf{n} \times \boldsymbol{\eta})\right\rangle_{F}=\left\langle\mathbf{H}_{h}, \boldsymbol{\eta}^{t}\right\rangle_{F} .
$$

Furthermore, $\boldsymbol{\eta} \in \mathbf{M}_{\mathrm{h}}$ then we have $\left.(\boldsymbol{\eta} \cdot \mathbf{n})\right|_{\mathrm{F}}=0$ and

$$
\left\langle-\mathbf{n} \times\left(\mathbf{n} \times \mathbf{H}_{h}\right), \boldsymbol{\eta}\right\rangle_{F}=\left\langle\mathbf{H}_{h}, \boldsymbol{\eta}\right\rangle_{F}, \quad \forall F \in \mathcal{F}_{h}, \quad \forall \boldsymbol{\eta} \in \mathbf{M}_{h} .
$$

The efficiency of the HDG implementation arises from obtaining an element-wise matrix system to construct a global system for the trace space degrees of freedom. Let $\underline{\Lambda}^{f}=\left[\underline{\Lambda}_{\mathrm{u}}^{\mathrm{f}}, \underline{\Lambda}_{\mathrm{w}}^{\mathrm{f}}\right]^{\mathrm{T}}$ denote the column vector of degrees of freedom on the face $\mathrm{F}_{\mathrm{f}} \in \mathcal{F}_{\mathrm{h}}$, of size $2 \mathrm{~N}_{\mathrm{F}}^{\mathrm{f}}$. We define the global trace vector of degrees of freedom, denoted by $\underline{\Lambda}$, as the concatenation of these vectors for all faces $\mathrm{F}_{\mathrm{f}}$ in $\mathcal{F}_{\mathrm{h}}$. The discretization of 17 and 18 eventually leads to the following linear system for the global trace $\underline{\Lambda}$

$$
\mathbb{K} \underline{\Lambda}=\underline{g} .
$$

where:

- $\mathbb{K}$ the matrix of size $\sum_{\mathrm{f}=1}^{\left|\mathcal{F}_{\mathrm{h}}\right|} 2 \mathrm{~N}_{\mathrm{F}}^{\mathrm{f}} \times \sum_{\mathrm{f}=1}^{\left|\mathcal{F}_{\mathrm{h}}\right|} 2 \mathrm{~N}_{\mathrm{F}}^{\mathrm{f}}$, defined by

$$
\mathbb{K}=\sum_{e=1}^{\left|\mathcal{T}_{h}\right|}\left[\mathcal{A}_{H D G}^{e}\right]^{T} \mathbb{K}^{e} \mathcal{A}_{H D G}^{e}=\sum_{e=1}^{\left|\mathcal{T}_{h}\right|}\left[\mathcal{A}_{H D G}^{e}\right]^{T}\left(\mathbb{G}^{e}-\mathbb{B}^{e}\left[\mathbb{A}^{e}\right]^{-1} \mathbb{C}^{e}\right) \mathcal{A}_{H D G}^{e}
$$

- $\underline{\mathrm{g}}$ the column vector $\sum_{\mathrm{f}=1}^{\left|\mathcal{F}_{\mathrm{h}}\right|} 2 \mathrm{~N}_{\mathrm{F}}^{\mathrm{f}}$, defined by $\underline{\mathrm{g}}=\sum_{\mathrm{e}=1}^{\left|\mathcal{T}_{\mathrm{h}}\right|}\left[\mathcal{A}_{\mathrm{HDG}}^{\mathrm{e}}\right]^{\top} \underline{\mathrm{g}}^{\mathrm{e}}$.

In the above expressions we have used the trace spreading operator $\mathcal{A}_{\mathrm{HDG}}$ which spreads or scatters the unique trace space values to their local face vectors. We can represent the operator $\mathcal{A}_{\mathrm{HDG}}$ as a matrix of size

$$
\sum_{e=1}^{\left|\mathcal{T}_{h}\right|}\left(\sum_{l=1}^{\left|\nu_{e}\right|} 2 N_{F}^{\sigma(e, l)}\right) \times \sum_{f=1}^{\left|\mathcal{F}_{h}\right|} 2 N_{F}^{f}
$$


Furthermore we organize this matrix by elements such that

$$
\mathcal{A}_{H D G}=\left[\mathcal{A}_{H D G}^{e_{1}}, \cdots, \mathcal{A}_{H D G}^{e_{\left|\mathcal{T}_{h}\right|}}\right]^{T},
$$

where the action of $\mathcal{A}_{\mathrm{HDG}}^{\mathrm{e}}$ is to copy global trace space information into local (elemental) storage. Then for each element $\mathrm{K}_{\mathrm{e}} \in \mathcal{T}_{\mathrm{h}}$ we define a matrix $\mathcal{A}_{\mathrm{HDG}}^{\mathrm{e}}$ of size

such that

$$
\sum_{l=1}^{\left|\nu_{e}\right|} 2 N_{F}^{\sigma(e, l)} \times \sum_{f=1}^{\left|\mathcal{F}_{h}\right|} 2 N_{F}^{f},
$$

$$
\mathcal{A}_{H D G}^{e} \underline{\Lambda}=\left[\underline{\Lambda}^{\sigma(e, 1)}, \cdots, \underline{\Lambda}^{\sigma\left(e,\left|\nu_{e}\right|\right)}\right]^{T}
$$

Remark 5. Let us assume that the interpolation degrees are $\mathrm{p}_{\mathrm{e}}=\mathrm{p}_{\mathrm{f}}=\mathrm{p}, \forall \mathrm{K}_{\mathrm{e}} \in \mathcal{T}_{\mathrm{h}}, \forall \mathrm{F}_{\mathrm{f}} \in \mathcal{F}_{\mathrm{h}}$. Then, when considering a continuous finite element (CFE) formulation based on Nedelec's first family of face/edge elements in a simplex (tetrahedron) for solving the three-dimensional frequency-domain Maxwell equations 10 , the total number of globally coupled degrees of freedom is $\frac{\mathrm{p}(\mathrm{p}+2)(\mathrm{p}+3)}{2}\left|\mathcal{T}_{\mathrm{h}}\right|$. The corresponding number for the HDG method is $(\mathrm{p}+1)(\mathrm{p}+2)\left|\mathcal{F}_{\mathrm{h}}\right|$. Since for a simplicial mesh $\left|\mathcal{F}_{\mathrm{h}}\right| \approx 2\left|\mathcal{T}_{\mathrm{h}}\right|$, the ratio of the globally coupled degrees of freedom is roughly $\frac{4(p+1)}{p(p+3)}$ for the HDG method over the FE method. We thus see that for $p=1$, we obtain that the HDG method requires two time more globally coupled degrees of freedom than CFE, while for $p>2$, this number is lower for the HDG method.

\section{5 | SOLUTION STRATEGIES}

For solving the sparse linear system 20 for the degrees of freedom of the hybrid variable $\boldsymbol{\Lambda}$, we adopt and compare in this study three strategies:

- A parallel LU factorization tailored for sparse matrices. Two sparse direct solvers have been considered: MUMPS (MUItifrontal Massively Parallel Sparse direct Solver) ${ }^{11}$ and PaStiX (Parallel Sparse matriX package) ${ }^{12}$. Both of theses solvers offer a hybrid MPI-thread parallel implementation;

- An algebraic hybrid iterative-direct solver whose design is based on domain decomposition principles: MaPHyS (Massively Parallel Hybrid Solver) 13 ;

- A PDE-based Schwarz algorithm based on transmission conditions at subdomain interfaces that are compliant with the intrinsic characteristics of the system of Maxwell equations 2 - -9 .

We refer to the above-mentioned references for more detail about these solution strategies and we briefly summarize below the main principles

and features of the two domain decomposition based solvers into the two next subsections.

\section{1 | The MaPHyS solver}

The solution of large sparse linear systems is a critical operation for many numerical simulations. To cope with the hierarchical design of modern supercomputer architectures, hybrid solvers based on algebraic domain decomposition methods have been proposed. Among them, approaches consisting of solving the (local) problem on the interior of the subdomains with a sparse direct method and the (global) problem on their interface with a preconditioned iterative method applied to the related Schur Complement have shown an attractive potential as they can combine both the robustness of direct methods and the low memory footprint of iterative methods. MaPHyS ${ }^{13}$ is a parallel linear solver implementing this idea.

One of the drawbacks of such a hybrid method is the number of iterations to convergence for the iterative part that increases with the total number of subdomains. This numerical scalability issue with domain decomposition solvers is very well known (see 14 
for example). To tackle this issue, MaPHyS proposes two solutions. The first one is an algebraic coarse grid correction mechanism for symmetric positive definite systems allowing to keep constant the number of iterations to convergence when the number of subdomains is increased. However it cannot be exploited here in its current design because the matrix operator of the HDG hybrid variable system does not have the required mathematical property. Further investigation in that direction is required and is the subject of an on going work. The second solution, which is employable for this study, is to exploit two levels of parallelism (MPI parallelism between the subdomains and thread parallelism within each subdomain). This allows to limit the overall number of subdomains when addressing a large number of cores, enabling to limit the number of required iterations to reach convergence (see 15 for more details).

The main advantage of the MaPHyS solver is that it is a black-box numerical tool and, as such, it can theoretically be applied to any matrix system independently of the nature of the underlying PDE model. However, the main weakness of its approach is its possible lack of numerical efficiency; in a PDE framework the preconditioner can be interpreted as the solution of a Dirichlet problem on a larger domain (i.e., at the boundary of the neighboring domains); It is appropriated for PDE with a smooth decay of the kernel but it is far from optimal from our the PDE characteristics point of view. Devising a robust (and scalable) algebraic domain decomposition solver is still considered as a challenging research topic by the numerical linear algebra community.

\section{2 | The continuous Schwarz algorithm}

Alternatively to a purely algebraic approach such as the one adopted in MaPHyS, one can design a Schwarz algorithm at the continuous PDE level, which is then discretized using one of several possible finite element schemes including DG-type schemes. In 2 we proposed such an approach in the form of a Schwarz algorithm where Després type conditions ${ }^{16}$ are imposed at the interfaces between neighboring subdomains. These conditions actually translate into a continuity condition for the incoming characteristic variables in the case of the first-order Maxwell system.

To simplify the presentation we assume a two-subdomain decomposition of the domain $\Omega$ into overlapping or non-overlapping subdomains $\Omega_{1}$ and $\Omega_{2}$. We set $\Gamma_{1,2}=\partial \Omega_{1} \cap \bar{\Omega}_{2}$ and $\Gamma_{2,1}=\partial \Omega_{2} \cap \bar{\Omega}_{1}$. By $\mathbf{n}_{i, j}(i, j \in\{1,2\})$, we denote the outward unit normal vector to the interface $\Gamma_{i, j}$. The classical Schwarz algorithm in this setting is an iterative method where the $(n+1)$-th iterate is defined from the $\mathrm{n}$-th iterate by solving the subdomain problems

$$
\begin{aligned}
& \left\{\begin{array}{l}
\mathrm{i} \omega \varepsilon_{\mathrm{r}} \mathbf{E}^{(1), \mathrm{n}+1}-\operatorname{curl} \mathbf{H}^{(1), \mathrm{n}+1}=-\mathbf{J} \text { in } \Omega_{1}, \\
\mathrm{i} \omega \mu_{\mathrm{r}} \mathbf{H}^{(1), \mathrm{n}+1}+\operatorname{curl} \mathbf{E}^{(1), \mathrm{n}+1}=0 \text { in } \Omega_{1}, \\
\mathcal{B}_{\mathbf{n}_{1,2}}\left(\mathbf{E}^{(1), \mathrm{n}+1}, \mathbf{H}^{(1), \mathrm{n}+1}\right)=\mathcal{B}_{\mathbf{n}_{1,2}}\left(\mathbf{E}^{(2), \mathrm{n}}, \mathbf{H}^{(2), \mathrm{n}}\right), \\
+ \text { Boundary conditions on } \partial \Omega_{1} \cap \partial \Omega,
\end{array}\right. \\
& \left\{\begin{array}{l}
\mathrm{i} \omega \varepsilon_{\mathrm{r}} \mathbf{E}^{(2), \mathrm{n}+1}-\mathbf{c u r l} \mathbf{H}^{(2), \mathrm{n}+1}=-\mathbf{J} \text { in } \Omega_{2}, \\
\mathrm{i} \omega \mu_{\mathrm{r}} \mathbf{H}^{(2), \mathrm{n}+1}+\operatorname{curl} \mathbf{E}^{(2), \mathrm{n}+1}=0 \text { in } \Omega_{2}, \\
\mathcal{B}_{\mathbf{n}_{2,1}}\left(\mathbf{E}^{(2), \mathrm{n}+1}, \mathbf{H}^{(2), \mathrm{n}+1}\right)=\mathcal{B}_{\mathbf{n}_{2,1}}\left(\mathbf{E}^{(1), \mathrm{n}}, \mathbf{H}^{(1), \mathrm{n}}\right), \\
+ \text { Boundary conditions on } \partial \Omega_{2} \cap \partial \Omega,
\end{array}\right.
\end{aligned}
$$

where $\left(\mathbf{E}^{(\mathrm{i}), \mathrm{n}}, \mathbf{H}^{(\mathrm{i}), \mathrm{n}}\right)$ denotes the electromagnetic field in the subdomain $\Omega_{\mathrm{i}}$ and this local electromagnetic field should converge towards the true electromagnetic field in $\Omega_{\mathrm{i}}$ as $\mathrm{n}$ goes to infinity. Moreover, $\mathcal{B}_{\mathbf{n}}$ denotes the transmission operator which, in the case of the classical Schwarz algorithm for the system of Maxwell's equations ${ }^{17}$ writes as

$$
\mathcal{B}_{\mathbf{n}}(\mathbf{E}, \mathbf{H})=\frac{1}{Z_{r}}(\mathbf{n} \times \mathbf{E})+\mathbf{n} \times(\mathbf{n} \times \mathbf{H})=\frac{1}{Z_{r}}(\mathbf{n} \times \mathbf{E})-\mathbf{H}^{t}
$$

with $Z_{r}=\sqrt{\frac{\mu_{r}}{\varepsilon_{r}}}$ being the impedance. The transmission conditions 22 are adapted to the characteristics of the system of Maxwell equations. In particular, they insure that the subdomain problems in the Schwarz algorithm are well-posed. Higher order optimized conditions have been proposed and studied in 17 , which are aiming to mitigate the effect of a reduction of the space discretization step on the convergence of the algorithm. However, these conditions do not deal with the dependence of the convergence on 
the number of subdomains. This approach has been adapted to the HDG discretization framework in ${ }^{9}$ but its implementation and numerical evaluation was limited to the case of a first order interpolation of the components of the electromagnetic field. In the present work, we have extended this PDE-based domain decomposition (DD) approach to an arbitrary high order HDG discretization method. We recall here the main features of the DD-HDG hybrid iterative-direct parallel solution strategy and refer to 9 for further details.

In the two-domain case, the discrete DD-HDG method is characterized by a reduced linear system

$$
\left(\begin{array}{ccc}
\mathrm{K}_{\mathrm{ii}}^{(1)} & 0 & \mathrm{~K}_{\mathrm{ig}}^{(1)} \\
0 & \mathrm{~K}_{\mathrm{ii}}^{(2)} & \mathrm{K}_{\mathrm{ig}}^{(2)} \\
\mathrm{K}_{\mathrm{gi}}^{(1)} & \mathrm{K}_{\mathrm{gi}}^{(2)} & \mathrm{K}_{\mathrm{gg}}^{(1)}+\mathrm{K}_{\mathrm{gg}}^{(2)}
\end{array}\right)\left(\begin{array}{c}
\Lambda_{\mathrm{h}, \mathrm{i}}^{(1)} \\
\Lambda_{\mathrm{h}, \mathrm{i}}^{(2)} \\
\Lambda_{\mathrm{h}, \mathrm{g}}
\end{array}\right)=\left(\begin{array}{c}
\mathrm{b}_{\mathrm{h}, \mathrm{i}}^{(1)} \\
\mathrm{b}_{\mathrm{h}, \mathrm{i}}^{(2)} \\
\mathrm{b}_{\mathrm{h}, \mathrm{g}}^{(1)}+\mathrm{b}_{\mathrm{h}, \mathrm{g}}^{(2)}
\end{array}\right),
$$

where

$$
\left(\begin{array}{ll}
\mathrm{K}_{\mathrm{ii}}^{(\mathrm{I})} & \mathrm{K}_{\mathrm{ig}}^{(\mathrm{I})} \\
\mathrm{K}_{\mathrm{gi}}^{(\mathrm{I})} & \mathrm{K}_{\mathrm{gg}}^{(\mathrm{I})}
\end{array}\right),
$$

denotes the matrix assembled on $\Omega_{1}$ for I in $\{1,2\}$. The subscript $g$ indicates that the degrees of freedom for faces are on $\Gamma_{1,2}$, while the subscript $i$ refer to degrees of freedom attached to internal faces in $\Omega_{1}$ or $\Omega_{2}$ but not on $\Gamma_{1,2}$. Following the lines of the work 18 , we introduce $A^{(1)}$ and $A^{(2)}$ that are matrices such that $A^{(1)}+A^{(2)}$ is invertible. Then, there is only one pair of Lagrange multipliers $\left(\Sigma^{(1)}, \Sigma^{(2)}\right)$ such that the coupled problem

$$
\begin{aligned}
&\left(\begin{array}{cc}
\mathrm{K}_{\mathrm{ii}}^{(1)} & \mathrm{K}_{\mathrm{ig}}^{(1)} \\
\mathrm{K}_{\mathrm{gi}}^{(1)} & \mathrm{K}_{\mathrm{gg}}^{(1)}+\mathrm{A}^{(1)}
\end{array}\right)\left(\begin{array}{c}
\Lambda_{\mathrm{h}, \mathrm{i}}^{(1)} \\
\Lambda_{\mathrm{h}, \mathrm{g}}^{(1)}
\end{array}\right)=\left(\begin{array}{c}
\mathrm{b}_{\mathrm{h}, \mathrm{i}}^{(1)} \\
b_{\mathrm{h}, \mathrm{g}}^{(1)}+\Sigma^{(1)}
\end{array}\right), \\
&\left(\begin{array}{cc}
\mathrm{K}_{\mathrm{ii}}^{(2)} & \mathrm{K}_{\mathrm{ig}}^{(2)} \\
\mathrm{K}_{\mathrm{gi}}^{(2)} & \mathrm{K}_{\mathrm{gg}}^{(2)}+\mathrm{A}^{(2)}
\end{array}\right)\left(\begin{array}{c}
\Lambda_{\mathrm{h}, \mathrm{i}}^{(2)} \\
\Lambda_{\mathrm{h}, \mathrm{g}}^{(2)}
\end{array}\right)=\left(\begin{array}{c}
\mathrm{b}_{\mathrm{h}, \mathrm{i}}^{(2)} \\
b_{\mathrm{h}, \mathrm{g}}^{(2)}+\Sigma^{(2)}
\end{array}\right), \\
& \Sigma^{(1)}+\Sigma^{(2)}-\left(\mathrm{A}^{(1)}+\mathrm{A}^{(2)}\right) \Lambda_{\mathrm{h}, \mathrm{g}}^{(1)}=\Sigma^{(1)}+\Sigma^{(2)}-\left(\mathrm{A}^{(1)}+\mathrm{A}^{(2)}\right) \Lambda_{\mathrm{h}, \mathrm{g}}^{(2)}=0,
\end{aligned}
$$

is equivalent to problem 23. From [23, we are led to solve the following linear system for the interface variables $\Sigma^{(1)}$ and $\Sigma^{(2)}$

$$
\begin{array}{r}
\left(\begin{array}{cc}
\mathrm{I} & \mathrm{I}-\mathrm{A}^{(12)}\left(\mathrm{S}^{(2)}+\mathrm{A}^{(2)}\right)^{-1} \\
\mathbf{I}-\mathrm{A}^{(12)}\left(\mathrm{S}^{(1)}+\mathrm{A}^{(1)}\right)^{-1} & \mathrm{I}
\end{array}\right)\left(\begin{array}{c}
\Sigma^{(1)} \\
\Sigma^{(2)}
\end{array}\right)= \\
\left(\begin{array}{l}
\mathrm{A}^{(12)}\left(\mathrm{S}^{(2)}+\mathrm{A}^{(2)}\right)^{-1} \mathrm{c}_{\mathrm{h}, \mathrm{g}}^{(2)} \\
\mathrm{A}^{(12)}\left(\mathrm{S}^{(1)}+\mathrm{A}^{(1)}\right)^{-1} \mathrm{c}_{\mathrm{h}, \mathrm{g}}^{(1)}
\end{array}\right),
\end{array}
$$

with $\mathrm{A}^{(12)}=\mathrm{A}^{(1)}+\mathrm{A}^{(2)}$ and where

$$
S^{(\ell)}=K_{g g}^{(\ell)}-K_{g i}^{(\ell)}\left(K_{i i}^{(\ell)}\right)^{-1} K_{i g}^{(\ell)} \text { and } c_{h, g}^{(\ell)}=b_{h, g}^{(\ell)}-K_{g i}^{(\ell)}\left(K_{i i}^{(\ell)}\right)^{-1} b_{h, i}^{(\ell)}, \quad \ell \in\{1,2\} .
$$

In the present case, the matrix $\mathrm{A}^{(\ell)}$ is assembled by computing the following term

$$
\left(A^{(\ell)}\right)_{i, j}=-Z_{r}^{(\ell)}\left\langle\boldsymbol{\eta}_{j}, \boldsymbol{\eta}_{i}\right\rangle_{\Gamma_{1,2}}
$$

where $\left(\boldsymbol{\eta}_{\mathrm{i}}\right)_{\mathrm{i}}$ is a basis of $\mathbf{M}_{\mathrm{h}}^{\mathrm{p}}$ restricted to $\Gamma_{1,2}$. Thus $\mathrm{A}^{(1)}+\mathrm{A}^{(2)}$ is invertible if and only if $Z_{r}^{(1)}+Z_{r}^{(2)} \neq 0$, which is obviously the case here. The interface system 25 is then solved by a BiCGStab $(\ell)$ Krylov subspace method without applying a preconditioner, while the inverse of the subdomain operators $K_{i i}$ in 26 are computed using the MUMPS or PaStiX sparse direct solvers (see ${ }^{9}$ for algorithmic aspects). In practice, we have never experienced a convergence failure when solving the interface system with an unpreconditioned BiCGStab $(\ell)$ Krylov subspace method. 


\section{6 | RESULTS}

\section{1 | Computing platforms and simulation parameters}

For the numerical simulations reported below we have used the following computing systems:

- The Plafrim cluster at Inria Bordeaux - Sud-Ouest. Each node of this system is equipped with an Intel E5-2680 running at 2.5 $\mathrm{GHz}$, with 24 cores on each node and $128 \mathrm{~GB}$ RAM per node. The interconnection network is an InfiniBand QDR TrueScale $40 \mathrm{~GB} / \mathrm{s}$ switch.

- The Occigen Bull/Atos cluster at CINES. Each node of this system is equipped with an Intel E5-2690 running at 2.6 GHz, with 24 cores on each node and 64 GB or 128 GB RAM per node.

For the hybrid iterative-direct domain decomposition solvers, we adopt a convergence criterion of the form

$$
\max _{i=1, \ldots, \# s d} \frac{\left\|\left(\Lambda_{h, g}^{(i)}\right)^{k+1}-\left(\Lambda_{h, g}^{(i)}\right)^{k}\right\|}{\left\|\left(\Lambda_{h, g}^{(i)}\right)^{1}-\left(\Lambda_{h, g}^{(i)}\right)^{0}\right\|}<\eta,
$$

where $\left(\Lambda_{\mathrm{h}, \mathrm{g}}^{(\mathrm{i})}\right)^{\mathrm{k}}$ denotes the vector of degrees of freedom of $\Lambda$ located on the part of the boundary of subdomain $\Omega_{\mathrm{i}}$ which is shared with neighboring subdomains, resulting from the $\mathrm{k}$-th iteration of the domain decomposition solver. Moreover, the targeted convergence threshold is computed as $\eta=\mathrm{h}_{\mathrm{m}}^{\mathrm{P}_{\mathrm{M}}+2}$ with $\mathrm{h}_{\mathrm{m}}$ the minimum length of an edge of the mesh and $\mathrm{P}_{\mathrm{M}}$ the maximum interpolation degree since in the general case the interpolation degree is defined locally within each cell and face of the mesh.

\section{2 | Plane wave propagation in vacuum}

We first consider a (model) problem consisting in the propagation of a plane wave in vacuum. This problem, which has no physical relevance, has been chosen because of its simplicity, especially in view of performing a large number of simulations to assess various parameters such as the problem size by adjusting the resolution of the mesh and the interpolation order in the HDG formulation, and the parallelization modes (MPI only versus MPI with multithreading). Indeed, the computational domain is chosen to be the unit cube $\Omega=[0,1]^{3}$ and the Silver-Müller absorbing boundary condition is imposed on $\partial \Omega$. The electromagnetic parameters $\varepsilon_{\mathrm{r}}$ and $\mu_{\mathrm{r}}$ are set to be 1.0 everywhere. The frequency of the incident plane wave is set to $f=600 \mathrm{MHz}$, then the angular frequency is $\omega=2 \pi \mathrm{f}=12 \pi \times 10^{8} \mathrm{rad} \cdot \mathrm{s}^{-1}\left(\simeq 12.6 \mathrm{rad} \cdot \mathrm{m}^{-1}\right)$. Its polarization is such that

$$
\mathbf{k}=\left(\begin{array}{c}
\mathrm{k}_{\mathrm{x}} \\
0 \\
0
\end{array}\right), \quad \mathbf{E}=\left(\begin{array}{c}
0 \\
0 \\
\mathrm{E}_{\mathrm{z}}
\end{array}\right) \quad \text { and } \quad \mathbf{H}=\left(\begin{array}{c}
0 \\
\mathrm{H}_{\mathrm{y}} \\
0
\end{array}\right) \text {, }
$$

where the wave number is $\mathrm{k}_{\mathrm{x}}=\omega \sqrt{\varepsilon_{1} \mu_{1}}$. We denote by $\mathrm{c}_{1}=1 / \sqrt{\varepsilon_{1} \mu_{1}}$ the wave propagation speed in vacuum, the wavelength in vacuum is then $\lambda_{1}=\mathrm{c}_{1} / \mathrm{f} \simeq 0.50$. Finally, the penalty parameter $\tau$ is set to be 1.0 everywhere.

We first investigate the numerical convergence order of the HDG- $\mathbb{P}_{\mid}$methods $(I=1,2,3$ and 4$)$, as a way to validate its computer implementation. For that purpose, a series of regular tetrahedral meshes are employed, which divide the unit cube into $\left(n_{x}-1\right) \times$ $\left(n_{y}-1\right) \times\left(n_{z}-1\right)$ cubes, where $n_{x}, n_{y}$ and $n_{z}$ are the number of grid points on the edges of the unit cube; then each cube is divided into six tetrahedrons. Tab. 1 1 gives the characteristics of the meshes employed for numerical tests, denoted by M1, M2, M3 and M4, while Tab.2 gives the corresponding numbers of degrees of freedom (DoF) of the discrete global and trace systems. Tab. 2 summarizes the $\mathrm{L}^{2}$-norm error and convergence behaviors of the HDG- $\mathbb{P}_{\mid}$methods. We observe that the numerical convergence orders for both $\mathbf{E}$ and $\mathbf{H}$ are optimal, i.e., the HDG- $\mathbb{P}_{\mid}$methods converge with order I +1 in the $\mathrm{L}^{2}$-norm.

We now investigate the multithreading performance in combination with the MPI-based parallelization. For that purpose we consider mesh M4 that we divide in 8 to 32 subdomains. On the MPI parallelism side, one subdomain is mapped to one MPI process 3 Then we also vary the number of threads per process from 1 to 12 . This allows us to take performance measurements on a desired

\footnotetext{
${ }^{3}$ Subsequently, in this study, subdomain, MPI process and process terms are completely equivalent
} 
TABLE 1 Plane wave propagation in vacuum: characteristics on uniform tetrahedral meshes used for numerical convergence analysis.

\begin{tabular}{rrrrrr}
\hline & $\left(\mathrm{n}_{\mathrm{x}}, \mathrm{n}_{\mathrm{y}}, \mathrm{n}_{\mathrm{z}}\right)$ & \# vertices & \# elements & \# faces & $\mathrm{h}$ \\
\hline M1 & $(13,7,7)$ & 637 & 2,692 & 5,544 & 0.2500 \\
M2 & $(17,9,9)$ & 1,377 & 6,144 & 12,928 & 0.1875 \\
M3 & $(21,11,11)$ & 2,541 & 12,000 & 25,000 & 0.1500 \\
M4 & $(25,13,13)$ & 4,225 & 20,736 & 42,912 & 0.1250 \\
\hline
\end{tabular}

TABLE 2 Plane wave propagation in vacuum: number of degrees of freedom (DoF) of the discrete global and trace systems.

\begin{tabular}{crr}
\hline HDG method & \# DoF $\Lambda$ field & \# DoF EM field \\
\hline HDG- $\mathbb{P}_{1}$ & 257,472 & 497,664 \\
HDG- $\mathbb{P}_{2}$ & 514,944 & $1,244,160$ \\
HDG- $\mathbb{P}_{3}$ & 858,240 & $2,488,320$ \\
HDG- $\mathbb{P}_{4}$ & $1,287,360$ & $4,354,560$ \\
\hline
\end{tabular}

\#core (where \# denotes number of) with different (\#thread,\#process) configurations, with the relation \#core =\#thread $\times$ \#process. We present performance results obtained for the following solutions strategies:

- MUMPS as global sparse direct solver;

- MaPHyS with either MUMPS or PaStiX as a subdomain solver;

- PDE-based Schwarz algorithm accelerated by a BiCGStab( $\ell$ ) Krylov subspace method with $\ell=6$ and MUMPS as a subdomain solver.

Simulations are run on the Plafrim cluster. Performance results are given in Figs. 2 and 3 for a strong scalability analysis of the various solution strategies. We evaluate different (\#thread,\#process) configurations. In all cases, the PDE-based Schwarz algorithm gives the lowest time to solution as it is clearly observed in Fig. 3 on which we plot the elapsed time for the best (\#thread,\#process) for a given total number of cores. In general, the MaPHyS algebraic domain decomposition solver scales almost ideally (the ideal speedup is given by the dashed line in Fig. 3. Regarding the solution based on a global sparse direct solver, namely MUMPS, we observe that the efficiency of the parallelization decreases as the number of cores increases. This is due to the granularity that becomes too fine to take benefit of the direct solver's solution strategy. However, it is somewhat competitive with MaPHyS from the time to solution point view, for the highest interpolation orders. We also observe that, for a given total number of cores, the best (\#thread,\#process) configuration is not the same for all the solution strategies.

\subsection{Scattering of a plane wave by an aircraft}

We now consider a more realistic application in the form of the scattering of plane wave by an aircrfat geometry. We start by considering a moderate size problem in order to evaluate the influence of the interpolation order on the accuracy of the HDG solution 
TABLE 3 Plane wave propagation in vacuum: numerical convergence analysis.

\begin{tabular}{|c|c|c|c|c|}
\hline \multirow[b]{2}{*}{$\mathbb{P}_{1}$} & \multicolumn{2}{|c|}{$\left\|\mathbf{E}-\mathbf{E}_{\mathrm{h}}\right\|_{2}$} & \multicolumn{2}{|c|}{$\left\|\mathbf{H}-\mathbf{H}_{\mathrm{h}}\right\|_{2}$} \\
\hline & Error & Order & Error & Order \\
\hline M1 & $7.10 \mathrm{e}^{-02}$ & - & $7.20 e^{-02}$ & - \\
\hline M2 & $4.27 e^{-02}$ & 1.8 & $4.29 \mathrm{e}^{-02}$ & 1.8 \\
\hline M3 & $2.85 e^{-02}$ & 1.8 & $2.85 e^{-02}$ & 1.8 \\
\hline \multirow[t]{2}{*}{ M4 } & $2.03 e^{-02}$ & 1.9 & $2.03 e^{-02}$ & 1.9 \\
\hline & \multicolumn{2}{|c|}{$\left\|\mathbf{E}-\mathbf{E}_{\mathrm{h}}\right\|_{2}$} & \multicolumn{2}{|c|}{$\left\|\mathbf{H}-\mathbf{H}_{\mathrm{h}}\right\|_{2}$} \\
\hline $\mathbb{P}_{3}$ & Error & Order & Error & Order \\
\hline M1 & $3.89 e^{-04}$ & - & $3.94 e^{-04}$ & - \\
\hline M2 & $1.24 \mathrm{e}^{-04}$ & 4.0 & $1.25 \mathrm{e}^{-04}$ & 4.0 \\
\hline M3 & $5.09 e^{-05}$ & 4.0 & $5.13 e^{-05}$ & 4.0 \\
\hline M4 & $2.46 \mathrm{e}^{-05}$ & 4.0 & $2.47 e^{-05}$ & 4.0 \\
\hline
\end{tabular}

\begin{tabular}{lrrrr}
\hline & \multicolumn{2}{c}{$\left\|\mathbf{E}-\mathbf{E}_{\mathrm{h}}\right\|_{2}$} & \multicolumn{2}{c}{$\left\|\mathbf{H}-\mathbf{H}_{\mathrm{h}}\right\|_{2}$} \\
$\mathbb{P}_{2}$ & \multicolumn{1}{c}{ Error } & Order & \multicolumn{1}{c}{ Error } & Order \\
\hline M1 & $6.78 \mathrm{e}^{-03}$ & - & $6.83 \mathrm{e}^{-03}$ & - \\
M2 & $2.90 \mathrm{e}^{-03}$ & 2.9 & $2.91 \mathrm{e}^{-03}$ & 3.0 \\
M3 & $1.49 \mathrm{e}^{-03}$ & 3.0 & $1.50 \mathrm{e}^{-03}$ & 3.0 \\
M4 & $8.68 \mathrm{e}^{-04}$ & 3.0 & $8.69 \mathrm{e}^{-04}$ & 3.0 \\
\hline & & & & \\
\hline & \multicolumn{1}{c}{$\left\|\mathbf{E}-\mathbf{E}_{\mathrm{h}}\right\|_{2}$} & & & \\
& & & & \\
$\mathbb{P}_{4}$ & Error & Order & Error & Order \\
\hline \multirow{2}{*}{ M1 } & $2.05 \mathrm{e}^{-05}$ & - & $2.07 \mathrm{e}^{-05}$ & - \\
M2 & $4.89 \mathrm{e}^{-06}$ & 5.0 & $4.94 \mathrm{e}^{-06}$ & 5.0 \\
M3 & $1.61 \mathrm{e}^{-06}$ & 5.0 & $1.62 \mathrm{e}^{-06}$ & 5.0 \\
M4 & $6.48 \mathrm{e}^{-07}$ & 5.0 & $6.52 \mathrm{e}^{-07}$ & 5.0 \\
\hline
\end{tabular}

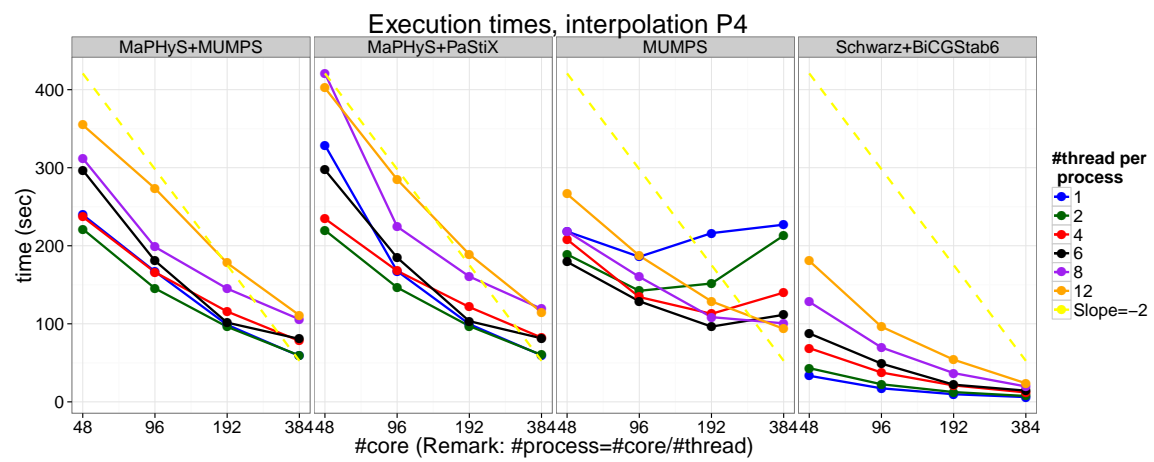

FIGURE 2 Plane wave propagation in vacuum: Strong scalability analysis of simulations run on the Plafrim cluster. On each plot, the various figures correspond to different configuration of subdomain partitioning (MPI parallelization) and threads within each subdomain.

for a given frequency of the incident plane wave. We also include a first assessment of the strong scalability when considering the MaPHyS solver on one hand, and the PDE-based Schwarz solver on the other hand. In a second step, we consider a more challenging setting and demonstrate the capabilities of the HDG method combined with the PDE-based Schwarz solver for simulating a problem involving more that one billion degrees of freedom for the discrete electromagnetic field. 

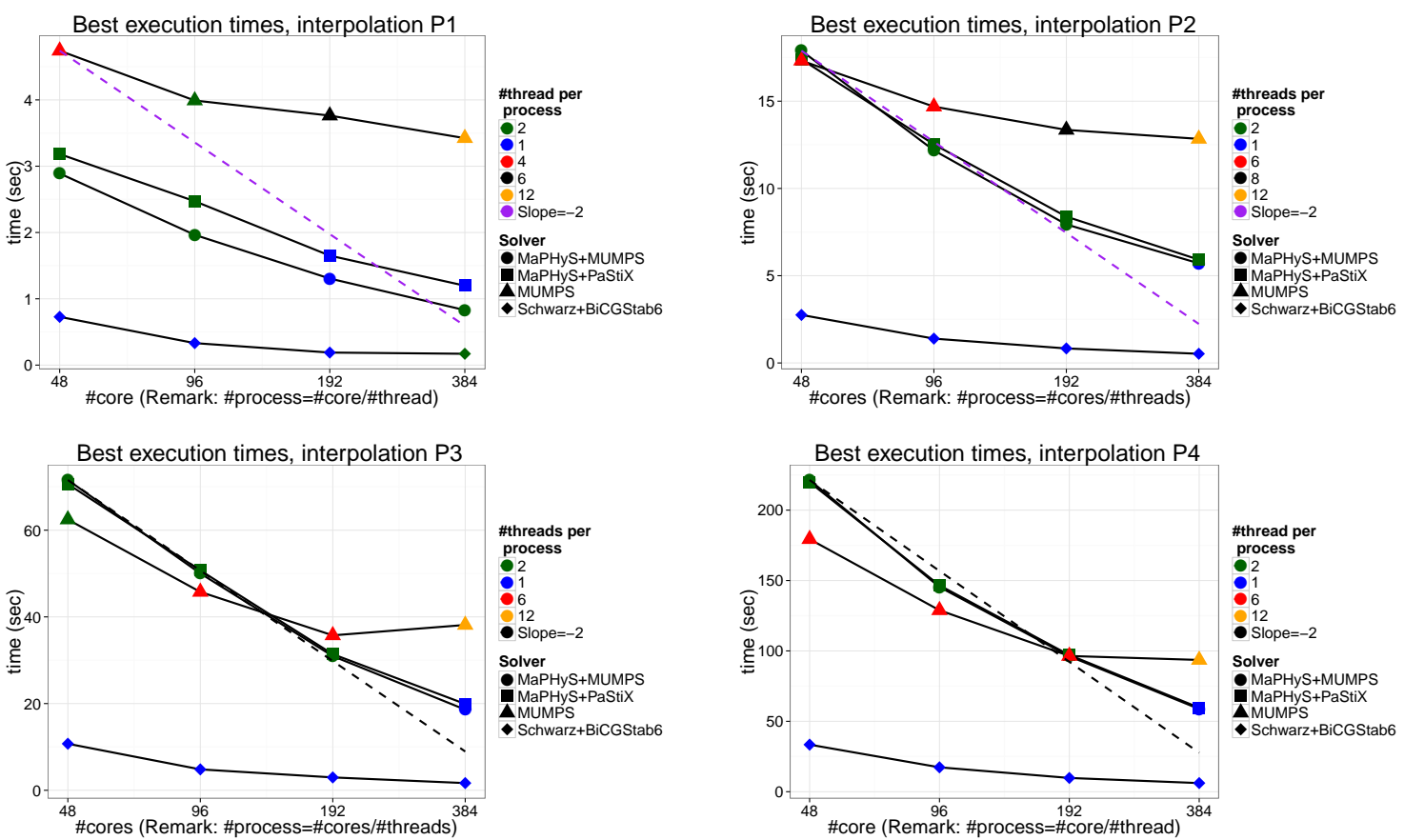

FIGURE 3 Plane wave propagation in vacuum: Strong scalability analysis of simulations run on the Plafrim cluster. On each plot, the various figures correspond to different configuration of subdomain partitioning (MPI parallelization) and threads within each subdomain. Here we show the best configurations in terms of number of subdomains and number of threads for a given total number of cores.

\subsubsection{Acuracy assessment with a uniform interpolation order}

For this series of numerical experiments, the computational domain is defined as the volume between the surface of a single aircraft and an artificial external boundary on which the absorbing boundary condition is applied (see Eq. 2]). The corresponding mesh consists of 1,645,874 elements and 3,521,251 faces. The frequency of the incident plane wave is fixed to $600 \mathrm{MHz}$. In Fig. $4 \mathrm{we}$ show physical solutions obtained with the HDG- $\mathbb{P}_{\ell}$ method for increasing values of the polynomial interpolation order $\ell$. A converged solution is obtained when using a third order approximation of the electromagnetic field components (for this particular mesh resolution). We observe here one advantage of increasing the interpolation order when the underlying mesh is relatively coarse. We however note that taking full benefit of higher order interpolation would also require an appropriate treatment of curved boundaries as it has been demonstrated in $\sqrt{5}$ for a similar HDG method in the two-dimensional case, or in 19 in the framework of a DG method for the three-dimensional time-domain Maxwell equations. Such an accurate handling of curved geometries in the present HDG setting will be the subject of a future work. The size, in terms of numbers of DoF, for the discrete hybrid variable and electromagnetic field components are summarized in Tab. 4 for several polynomial interpolation orders in the HDG method. We present results of simulations performed using MPI parallelization mode only (combined MPI and multithreaded parallel execution mode will be considered in the future for larger problem sizes). These simulations have been performed on the Occigen system. Performance figures (strong scalability analysis) are given in Tabs 6 and 7 We observe that the PDE-based Schwarz algorithm is the most efficient, and is scalable despite the increase of the number of iterations to convergence when increasing the number of subdomains 
for a given HDG- $\mathbb{P}_{\mathrm{p}}$ method. In the present context, the MaPHyS solver does not achieve the targeted accuracy threshold for the discrete HDG system based on second and third (not shown here) order polynomial interpolation. This is the main cause of scalability degradation between 768 and 1536 cores with the HDG- $\mathbb{P}_{1}$ method.

TABLE 4 Scattering of a plane wave by the Lockheed F-104 Starfighter: number of degrees of freedom (DoF) of the discrete global and trace systems.

\begin{tabular}{crr}
\hline HDG method & \# DoF $\Lambda$ field & \# DoF EM field \\
\hline HDG- $\mathbb{P}_{1}$ & $21,127,506$ & $39,500,976$ \\
HDG- $\mathbb{P}_{2}$ & $42,255,012$ & $98,752,440$ \\
HDG- $\mathbb{P}_{3}$ & $70,425,020$ & $197,504,880$
\end{tabular}

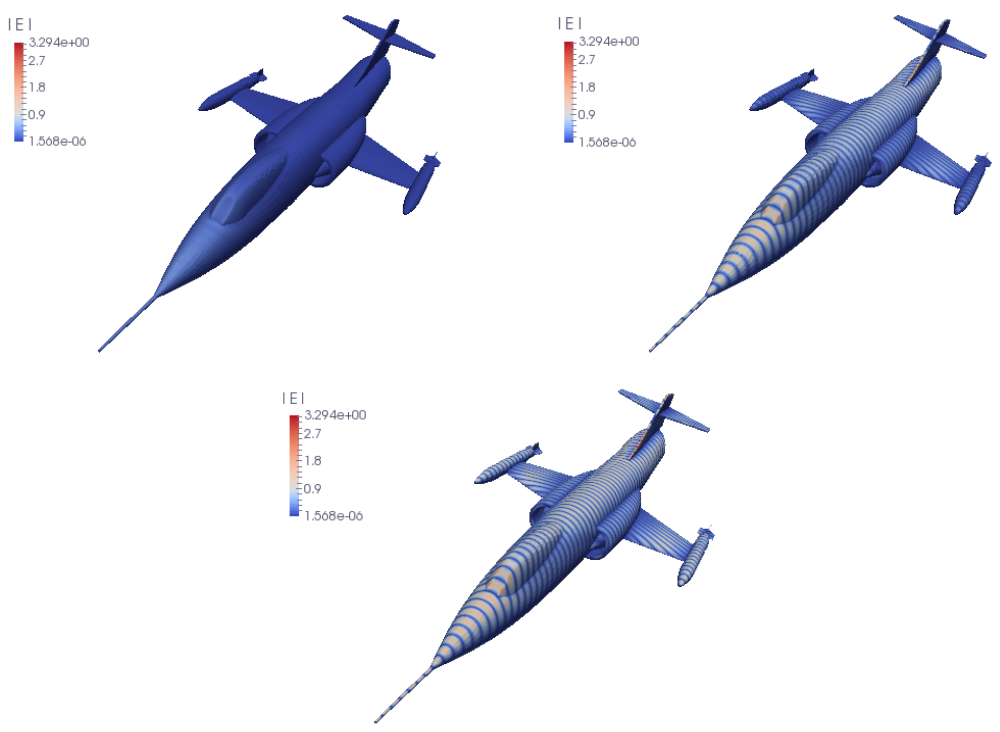

FIGURE 4 Scattering of a plane wave by the Lockheed F-104 Starfighter: contour line of $|\mathbf{E}|$ for the HDG- $\mathbb{P}_{1}$ (top left), HDG- $\mathbb{P}_{2}$ (top right) and HDG- $\mathbb{P}_{3}$ (bottom) methods.

\subsection{2 | Strong scaling for a large problem}

In order to further evaluate the strong scalability of the HDG method combined with the PDE-based Schwarz solver, we now consider a more challenging problem that consists in the scattering of a plane wave by a squadron of four Lockheed F-104 Starfighter. The constructed tetrahedral mesh contains $8,539,215$ elements and $18,045,563$. The frequency of the incident plane wave is agian 
fixed to $600 \mathrm{MHz}$. In Fig. 5 we show contour line of $|\mathrm{E}|$ for the HDG- $\mathbb{P}_{3}$ method. Simulations have been performed on the Occigen system. Strong scalability results are summarized in Tab. 8. Unfortunately, we have not been able to perform a simulation with 6144 cores when using the HDG- $\mathbb{P}_{2}$ and HDG- $\mathbb{P}_{3}$ methods, in order to have a more complete picture of the parallel performances. When considering the HDG- $\mathbb{P}_{1}$ method, the total problem size is not sufficient to obtain an optimal speedup when doubling the number of cores. In addition, the PDE-based Schwarz algorithm converges in a few iterations and the increase from 2 to 3 iterations is also impacting the achievable speedup. The situation is much better when increasing the interpolation order. A slight superlinear speedup is obtained for the simulations based on the HDG- $\mathbb{P}_{2}$ method, which can be attributed to the fact that as the number of cores, i.e., subdomains, is increased, the size and computational cost of the local $L$ and $U$ factors decrease non-linearly therefore resulting in a superlinear acceleration of the subdomain solves. Finally, we note that when using the HDG- $\mathbb{P}_{3}$ method, the corresponding one billion size problem is solved in less that $4 \mathrm{mn}$ on 3072 cores.

TABLE 5 Scattering of a plane wave by by a squadron of Lockheed F-104 Starfighter: number of degrees of freedom (DoF) of the discrete global and trace systems.

\begin{tabular}{crr}
\hline HDG method & \# DoF $\Lambda$ field & \# DoF EM field \\
\hline HDG- $\mathbb{P}_{1}$ & $108,273,378$ & $204,941,160$ \\
HDG- $\mathbb{P}_{2}$ & $216,546,756$ & $512,352,900$ \\
HDG- $\mathbb{P}_{3}$ & $360,911,260$ & $1,024,705,800$ \\
\hline
\end{tabular}

TABLE 6 Scattering of a plane wave by the Lockheed F-104 Starfighter: strong scalability analysis, Schwarz algorithm with PaStiX as a local solver.

\begin{tabular}{crrrrrr}
\hline HDG method & \# cores & \# iter & Fact. Time & Sol. Time & Wall time & Speedup \\
\hline HDG- $\mathbb{P}_{1}$ & 384 & 3 & $2.6 \mathrm{sec}$ & $3.7 \mathrm{sec}$ & $6.8 \mathrm{sec}$ & 1.00 \\
- & 768 & 4 & $0.8 \mathrm{sec}$ & $2.3 \mathrm{sec}$ & $3.4 \mathrm{sec}$ & 2.00 \\
HDG- $\mathbb{P}_{2}$ & 384 & 10 & $16.7 \mathrm{sec}$ & $40.5 \mathrm{sec}$ & $58.7 \mathrm{sec}$ & 1.00 \\
- & 768 & 12 & $5.1 \mathrm{sec}$ & $21.5 \mathrm{sec}$ & $27.1 \mathrm{sec}$ & 2.15 \\
HDG- $\mathbb{P}_{3}$ & 768 & 23 & $18.8 \mathrm{sec}$ & $102.1 \mathrm{sec}$ & $122.6 \mathrm{sec}$ & 1.00 \\
& 1536 & 26 & $5.1 \mathrm{sec}$ & $52.0 \mathrm{sec}$ & $58.7 \mathrm{sec}$ & 2.10 \\
\hline
\end{tabular}

\subsection{Electromagnetic wave propagation in head tissues}

Nowadays, numerical modeling is increasingly used and progressively becoming a mandatory path for the study of the interaction of electromagnetic fields with biological tissues. This is for instance the case for the evaluation of the distribution of the SAR 
TABLE 7 Scattering of a plane wave by the Lockheed F-104 Starfighter: strong scalability analysis, MaPHyS solver with PaStiX as a local solver.

\begin{tabular}{crrrrrrr}
\hline HDG method & \# cores & \# iter & Fact. Time & Prec. Time & Sol. Time & Wall time & Speedup \\
\hline HDG- $\mathbb{P}_{1}$ & 384 & 93 & $26.3 \mathrm{sec}$ & $23.5 \mathrm{sec}$ & $21.5 \mathrm{sec}$ & $73.4 \mathrm{sec}$ & 1.00 \\
- & 768 & 194 & $7.2 \mathrm{sec}$ & $8.1 \mathrm{sec}$ & $18.6 \mathrm{sec}$ & $34.7 \mathrm{sec}$ & 2.10 \\
- & 1536 & 374 & $2.3 \mathrm{sec}$ & $2.5 \mathrm{sec}$ & $18.1 \mathrm{sec}$ & $23.5 \mathrm{sec}$ & 3.15 \\
HDG- $\mathbb{P}_{2}$ & 768 & 5000 & $53.1 \mathrm{sec}$ & $55.2 \mathrm{sec}$ & $2353.0 \mathrm{sec}$ & $2463.0 \mathrm{sec}$ & 1.00 \\
- & 1536 & 5000 & $13.9 \mathrm{sec}$ & $16.4 \mathrm{sec}$ & $1393.0 \mathrm{sec}$ & $1426.0 \mathrm{sec}$ & 1.75 \\
\hline
\end{tabular}

TABLE 8 Scattering of a plane wave by a squadron of the Lockheed F-104 Starfighter: strong scalability analysis, Schwarz algorithm with PaStiX as a local solver.

\begin{tabular}{crrrrrr}
\hline HDG method & \# cores & \# iter & Fact. Time & Sol. Time & Wall time & Speedup \\
\hline HDG- $\mathbb{P}_{1}$ & 1536 & 2 & $4.4 \mathrm{sec}$ & $3.8 \mathrm{sec}$ & $9.0 \mathrm{sec}$ & 1.00 \\
- & 3072 & 3 & $1.7 \mathrm{sec}$ & $3.1 \mathrm{sec}$ & $5.1 \mathrm{sec}$ & 1.75 \\
HDG-P & 1536 & 14 & $30.0 \mathrm{sec}$ & $85.0 \mathrm{sec}$ & $115.0 \mathrm{sec}$ & 1.00 \\
- & 3072 & 15 & $8.9 \mathrm{sec}$ & $40.0 \mathrm{sec}$ & $49.9 \mathrm{sec}$ & 2.30 \\
HDG- $\mathbb{P}_{3}$ & 3072 & 28 & $34.0 \mathrm{sec}$ & $185.1 \mathrm{sec}$ & $221.6 \mathrm{sec}$ & 1.00 \\
\hline
\end{tabular}

(Specific Absorption Rate) which is a measure of the rate at which electric energy is absorbed by the tissues when exposed to a radiofrequency electromagnetic field. The SAR is defined as the power absorbed per mass of tissue and has units of watts per kilogram. It is usually averaged either over the whole body, or over a small sample volume (typically $1 \mathrm{~g}$ or $10 \mathrm{~g}$ of tissue). Such SAR calculations are at the basis of numerical dosimetry studies of the exposure of human tissues to microwave radiations from wireless communication systems 20,21_-22. These studies are useful for assessing the possible thermal effects (temperature rise in tissues resulting from electric energy dissipation) as well as for compliance testing to regulatory limits. Despite the high complexity both in terms of heterogeneity and geometrical features of tissues, the great majority of numerical dosimetry studies have been conducted using the FD (Finite Difference) method. In this method, the whole computational domain is discretized using a structured (Cartesian) grid. Due to the possible straightforward implementation of the algorithm and the availability of computational power, FD is currently the leading method for numerical assessment of human exposure to electromagnetic waves. However, the rather difficult departure from the commonly used Cartesian grid and cell size limitations regarding the discretization of very detailed structures of human tissues are often recognized as the main weaknesses of the method in this application context. The use of heterogenous model of human tissues based on unstructured meshes is particularly appealing since they allow for an accurate discretization of interfaces between different biological media, which are the localization of electromagnetic field discontinuities.

To illustrate this alternative modeling numerical dosimetry approach, we consider here a problem which is concerned with the simulation of the exposure of geometrical models of head tissues to an electromagnetic wave emitted by a localized source. Head tissues are segmented and the interfaces of a selected number of tissues (namely, the skin, the skull, the CSF - Cerebro Spinal Fluid and 

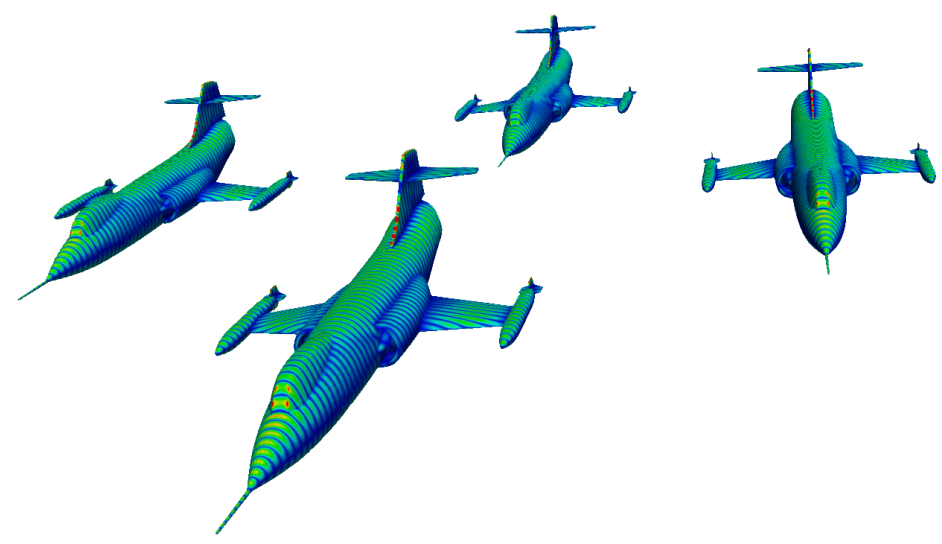

FIGURE 5 Scattering of a plane wave by a squadron of Lockheed F-104 Starfighter: contour line of $|\mathbf{E}|$ for the HDG- $\mathbb{P}_{3}$ method.

the brain) are triangulated. Then, these surface meshes are used as inputs for the generation of volume meshes. Note that the exterior of the head must also be meshed, up to a certain distance from the skin. The computational domain is here artificially bounded by a sphere on which the Silver-Müller condition is imposed. In the present case, the constructed geometrical model involves four tissues (skin, skull, CSF, brain) and we consider two global tetrahedral meshes: a coarse mesh (M1) with 366, 208 tetrahedra, 725, 136 triangular faces and a fine mesh (M2) with 1, 853, 832 tetrahedra, 3, 911, 256 triangular faces, see Fig. 6 and Tab.9. The electromagnetic parameters of the tissues are summarized in Tab. 10 where the values of the relative electrical permittivity correspond to a frequency $f=1800 \mathrm{MHz}$ and have been obtained from a special purpose online database. A dipolar type source is localized near the right ear of the head yielding a current of the form

$$
\mathbf{J}_{z}=z_{0} \delta\left(\mathbf{x}-\mathbf{x}^{s}\right),
$$

where $z_{0}$ is the free space intinsic impedance, $\delta$ is Dirac delta function and $\mathbf{x}^{s}=(-0.100,0.025,-0.015)$ the localization point of the source.
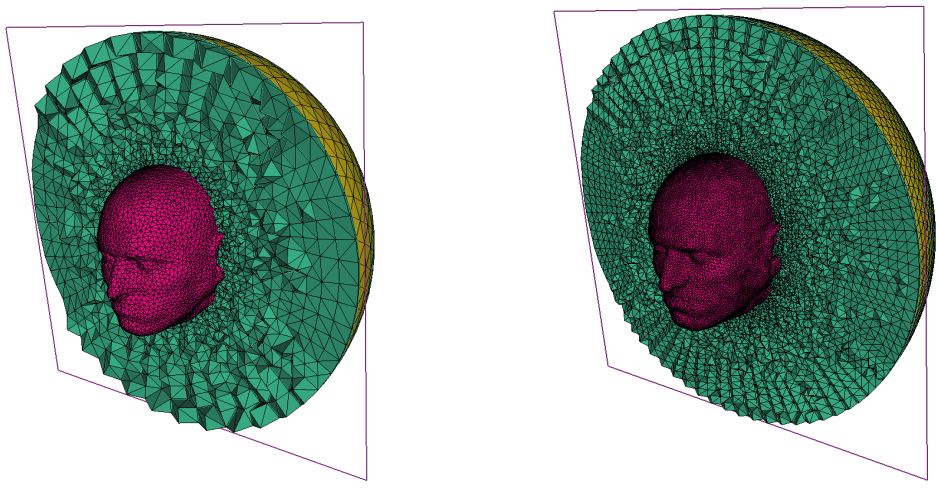

FIGURE 6 Exposure of head tissues to a localized source radiation: coarse/fine tetrahedral meshes (left/right). 


\begin{tabular}{lrrrrr}
\hline & \# Elements & \# Faces & $\mathrm{h}_{\min }(\mathrm{m})$ & $\mathrm{h}_{\max }(\mathrm{m})$ & $\mathrm{h}_{\max } / \mathrm{h}_{\min }$ \\
\hline Coarse mesh (M1) & 366,208 & 733,856 & $4.05 \mathrm{e}^{-4}$ & $4.54 \mathrm{e}^{-2}$ & 112 \\
Fine mesh (M2) & $1,853,832$ & $3,713,424$ & $1.59 \mathrm{e}^{-3}$ & $2.48 \mathrm{e}^{-2}$ & 15 \\
\hline
\end{tabular}

TABLE 9 Exposure of head tissues to a localized source radiation: characteristics of coarse and fine tetrahedral meshes.

\begin{tabular}{lrrrrr}
\hline & Vacuum & Skin & Skull & CSF & Brain \\
\hline$\varepsilon$ & 1.00 & 38.66 & 11.60 & 68.25 & 43.88 \\
$\sigma\left(\mathrm{S} \cdot \mathrm{m}^{-1}\right)$ & 0.00 & 1.18 & 0.27 & 2.28 & 0.97 \\
$\lambda(\mathrm{mm})$ & 166.67 & 26.79 & 48.90 & 20.16 & 25.14 \\
$\rho$ & 1.00 & $1,100.00$ & $1,200.00$ & $1,000.00$ & $1,050.00$ \\
\hline
\end{tabular}

TABLE 10 Exposure of head tissues to a localized source radiation: electromagnetic parameters of head tissues and vacuum.

\subsection{1 | SAR computations}

The number of degrees of freedom for the electromagnetic $(E M)$ and $\Lambda$ fields for a uniform $\mathbb{P}_{1}$ interpolation order are summarized in Tab. 11 for both meshes. The corresponding figures for a uniform $\mathbb{P}_{2}$ interpolation order are given in Tab. 12. Fig] 7 show the contour lines of the local SAR normalized by the maximal local SAR (in logarithmic scale) obtained with the HDG- $\mathbb{P}_{1}$ method. Fig reffig:SARP2 show the contour lines of the local SAR normalized by the maximal local SAR (in logarithmic scale) obtained with the HDG- $\mathbb{P}_{2}$ method. Note that the scale is the same for all pictures in Figs 7 and 8

\begin{tabular}{lrrrr}
\hline & \# Elements & \# Faces & \# DoFs EM & \# DoFs $\Lambda$ \\
\hline Coarse mesh (M1) & 366,208 & 733,856 & $8,788,992$ & $4,804,110$ \\
Fine mesh (M2) & $1,853,832$ & $3,713,424$ & $44,491,968$ & $23,467,536$ \\
\hline
\end{tabular}

TABLE 11 Exposure of head tissues to a localized source radiation: number of degrees of freedom for the electromagnetic (EM) and $\Lambda$ fields - HDG- $\mathbb{P}_{1}$ method.

\subsubsection{Acuracy and performance assessment with a non-uniform interpolation order}

In section 6.4.1 we have used a uniform interpolation order $\mathbb{P}_{1}$ or $\mathbb{P}_{2}$ in the simulations. Now, we illustrate the potential computational savings resulting from the use of a locally defined interpolation order. The strategy considered here is a first step towards the exploitation of p-adpativity relying on an a posteriori error estimator. Instead, we adopt here a very simple accuracy criterion. We could fix a minimum, maximum and average number of points per wavelength to decide upon the appropriate value of the interpolation order to be used in the simulation for a given unstructured tetrahedral mesh, as a function of $h_{\min }$ and $h_{\max }$, but this would not be very relevant because of the great disparity of size in the mesh from one element to another. For instance, in the coarse mesh the ratio $h_{\max } / h_{\min }$ is too high to exploit such a strategy, see Tab. 9 However, our implementation of the HDG method is able to work 

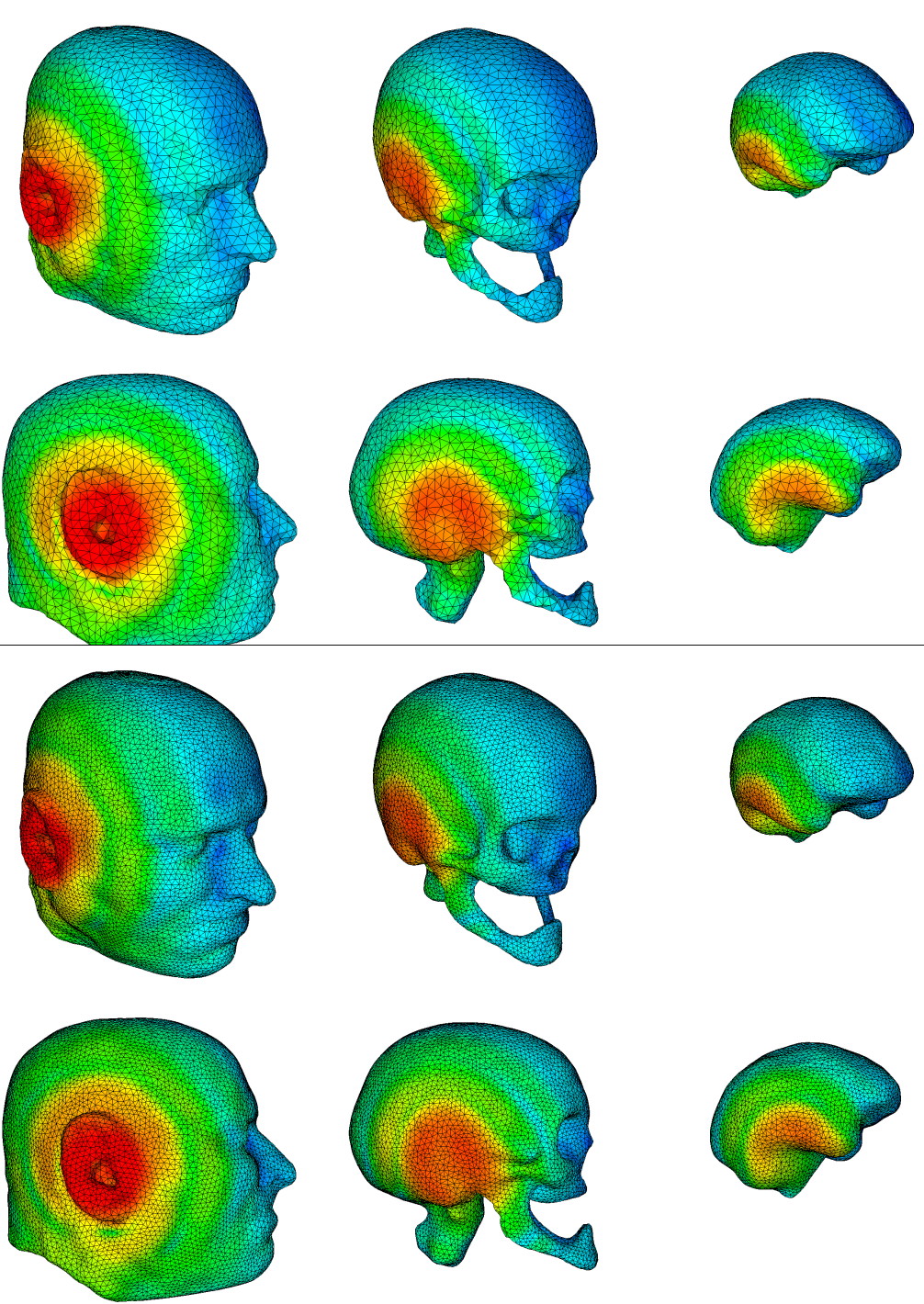

FIGURE 7 Exposure of head tissues to a localized source radiation: contour lines of the local SAR normalized by the maximal local SAR (logarithmic scale) on skin, skull and brain for the coarse mesh (top) and the fine mesh (bottom) - HDG- $\mathbb{P}_{1}$ results.

\begin{tabular}{lrrrr}
\hline & \# Elements & \# Faces & \#DoFs EM & \# DoFs $\boldsymbol{}$ \\
\hline Coarse mesh (M1) & 366,208 & 733,856 & $21,972,480$ & $9,608,220$ \\
Fine mesh (M2) & $1,853,832$ & $3,713,424$ & $111,229,920$ & $46,935,072$
\end{tabular}

TABLE 12 Exposure of head tissues to a localized source radiation: number degrees of freedom for the electromagnetic (EM) and $\boldsymbol{\Lambda}$ fields - HDG- $\mathbb{P}_{2}$ method. 

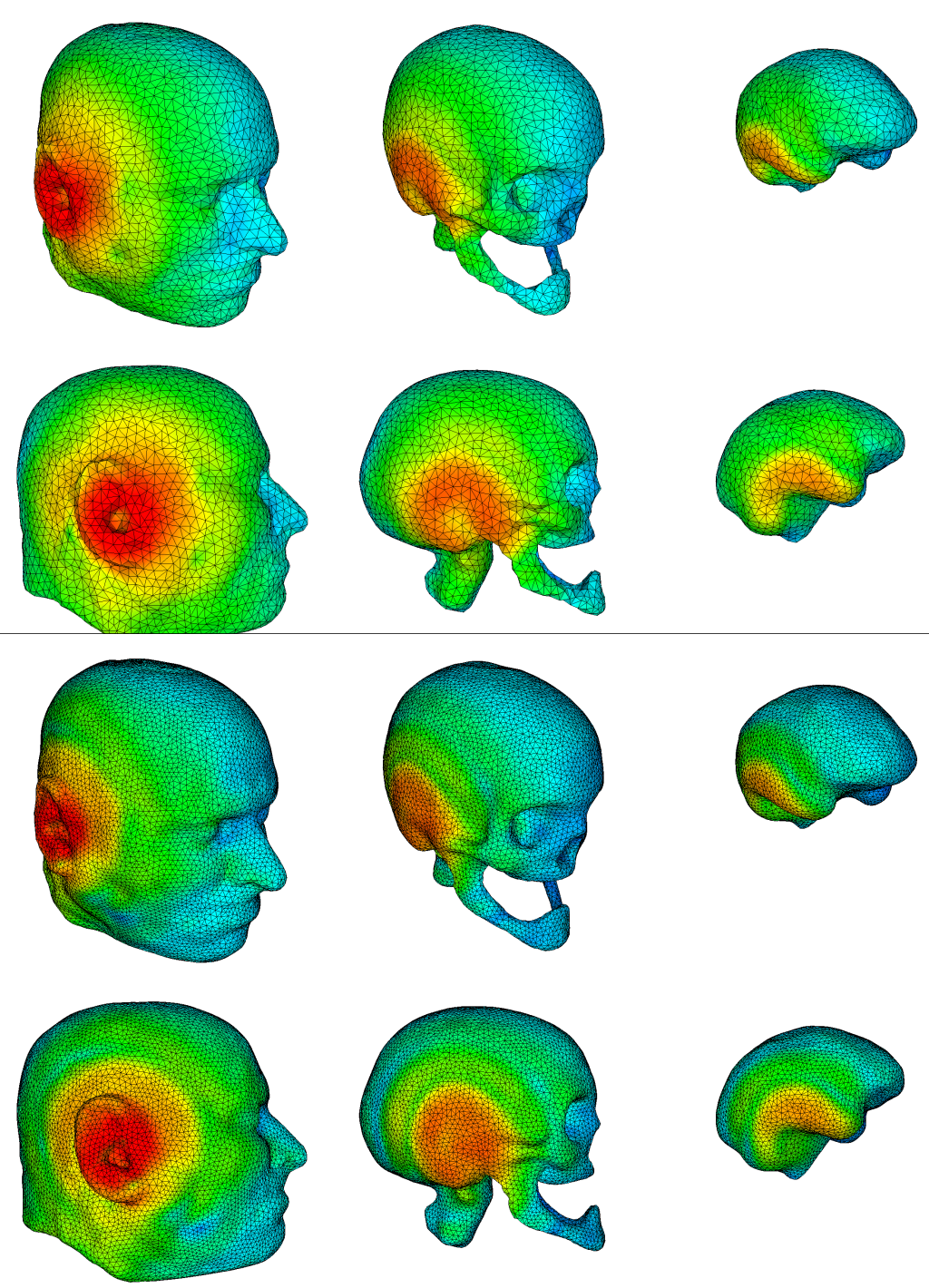

FIGURE 8 Exposure of head tissues to a localized source radiation: contour lines of the local SAR normalized by the maximal local SAR (logarithmic scale) on skin, skull and brain for the coarse mesh (top) and the fine mesh (bottom) - HDG- $\mathbb{P}_{2}$ results.

with a non-uniform (i.e., element-wise) interpolation order. This allows us to fix a minimum number of points per wavelength and adapt locally the interpolation order. This yields a discretization strategy that we refer to as HDG- $\mathbb{P}_{\text {local }}$ method.

We present numerical results obtained for the HDG- $\mathbb{P}_{\text {local }}$ method. We adopt an order distribution that allows us to obtain a minimum of 9 points per wavelength whatever the mesh under consideration. Note that the minimum of 9 points per wavelength has been fixed arbitrarily. The algorithm used for the order distribution in practice is described in Algo. 14 Once the interpolation order is fixed for each tetrahedron, the interpolation order for a triangular face is defined as the maximum between the orders in each element attached to this face. The interpolation order in the elements defines the number of degrees of freedom for the electromagnetic field while the interpolation order on faces define the number of degrees of freedom for the hybrid variable $\boldsymbol{\Lambda}$. The 
resulting order distribution, for a minimum of 9 points per wavelength, and the associated numbers of degrees of freedom for the electromagnetic and $\boldsymbol{\Lambda}$ fields are summarized in Tabi. 15 for both meshes.

All the simulations discussed in this section have been performed on the Occigen system using the PDE-based Schwarz algorithm. Peformance results for the HDG- $\mathbb{P}_{2}$ and HDG- $\mathbb{P}_{\text {ploc }}$ methods using mesh M2 are summarized in Tab. 13 These results clearly show the advantage of using a locally adapted inerpolation order strategy in terms of reduction of the overall wall clock time.

TABLE 13 Exposure of head tissues to a localized source radiation:: strong scalability analysis, Schwarz algorithm with PaStiX as a local solver.

\begin{tabular}{crrrrrr}
\hline HDG method & \# cores & \# iter & Fact. Time & Sol. Time & Wall time & Speedup \\
\hline HDG- $\mathbb{P}_{2}$ & 384 & 52 & $21.1 \mathrm{sec}$ & $255.6 \mathrm{sec}$ & $278.4 \mathrm{sec}$ & 1.00 \\
- & 768 & 65 & $6.5 \mathrm{sec}$ & $142.4 \mathrm{sec}$ & $149.6 \mathrm{sec}$ & 1.85 \\
- & 1536 & 78 & $2.5 \mathrm{sec}$ & $79.2 \mathrm{sec}$ & $82.4 \mathrm{sec}$ & 3.40 \\
\hline HDG- & 192 & 42 & $51.0 \mathrm{sec}$ & $288.1 \mathrm{sec}$ & $341.0 \mathrm{sec}$ & 1.00 \\
& 384 & 54 & $13.7 \mathrm{sec}$ & $159.6 \mathrm{sec}$ & $174.1 \mathrm{sec}$ & 1.95 \\
- & 768 & 60 & $4.5 \mathrm{sec}$ & $84.7 \mathrm{sec}$ & $89.6 \mathrm{sec}$ & 3.80 \\
- & 1536 & 74 & $1.6 \mathrm{sec}$ & $52.0 \mathrm{sec}$ & $53.9 \mathrm{sec}$ & 6.35 \\
\hline
\end{tabular}

Fig.9 summarizes the obtained results for HDG- $\mathbb{P}_{1}, \mathrm{HDG}-\mathbb{P}_{2}$ and HDG- $\mathbb{P}_{\text {local }}$ (9 points per wavelength) methods by representing the contour lines of the local SAR normalized by the maximal local SAR in selected planes for each method and mesh, on a single figure. We can observe that the results obtained for the uniform interpolation order $\mathbb{P}_{1}$ and the local interpolation orders for a minimum number of 9 points per wavelength are very close. Despite the fact that the minimum number of points per wavelength is 9 in both case, i.e., for the coarse and fine meshes, the results obtained for the coarse mesh seems to be slightly different from those obtained for the coarse mesh. This is due to the fact that the coarse mesh does not describe the geometrical model of head tissues with sufficient accuracy compared to the fine mesh. Note that this behavior seems in line with the order distribution, see Tab. 15

\section{7 | CONCLUSION}

In this paper, we have given an up-to-date picture of the development of a recently introduced hybridized DG formulation further adpated to the solution of the system of $3 \mathrm{~d}$ time-harmonic Maxwell equations. We have presented results from realistic $3 \mathrm{~d}$ simulations illustrating the capabilities of the proposed high order DD-HDG solver. We are currently working on several topics to enhance the capabilities of the proposed methodology. One of them is concerned with the possibility to adapt locally the interpolation order in the HDG method, based on an a posterori error estimator. Improving the numerical scalability and overall performance of the MaPHyS algebraic domain decomposition solver is also one of our main objectives in the short-term. Indeed, the current implementation of the Schwarz preconditioner in MaPHyS assumes classical Dirichlet-type interface conditions that are not adapted to matrix operators resulting from the discretization of the Maxwell equations. We thus consider the possibility of extending the $\mathrm{MaPHyS}$ approach by leveraging user-supplied discrete interface operators well suited to the underlying physical problem (i.e., in the present case, HDG-compliant forms of (22). Besides, a detailed assessment of the combined MPI+thread parallelism will allow to better characterize the best usage configurations for the MaPHyS algebraic solver, especially when simulating more realistic 


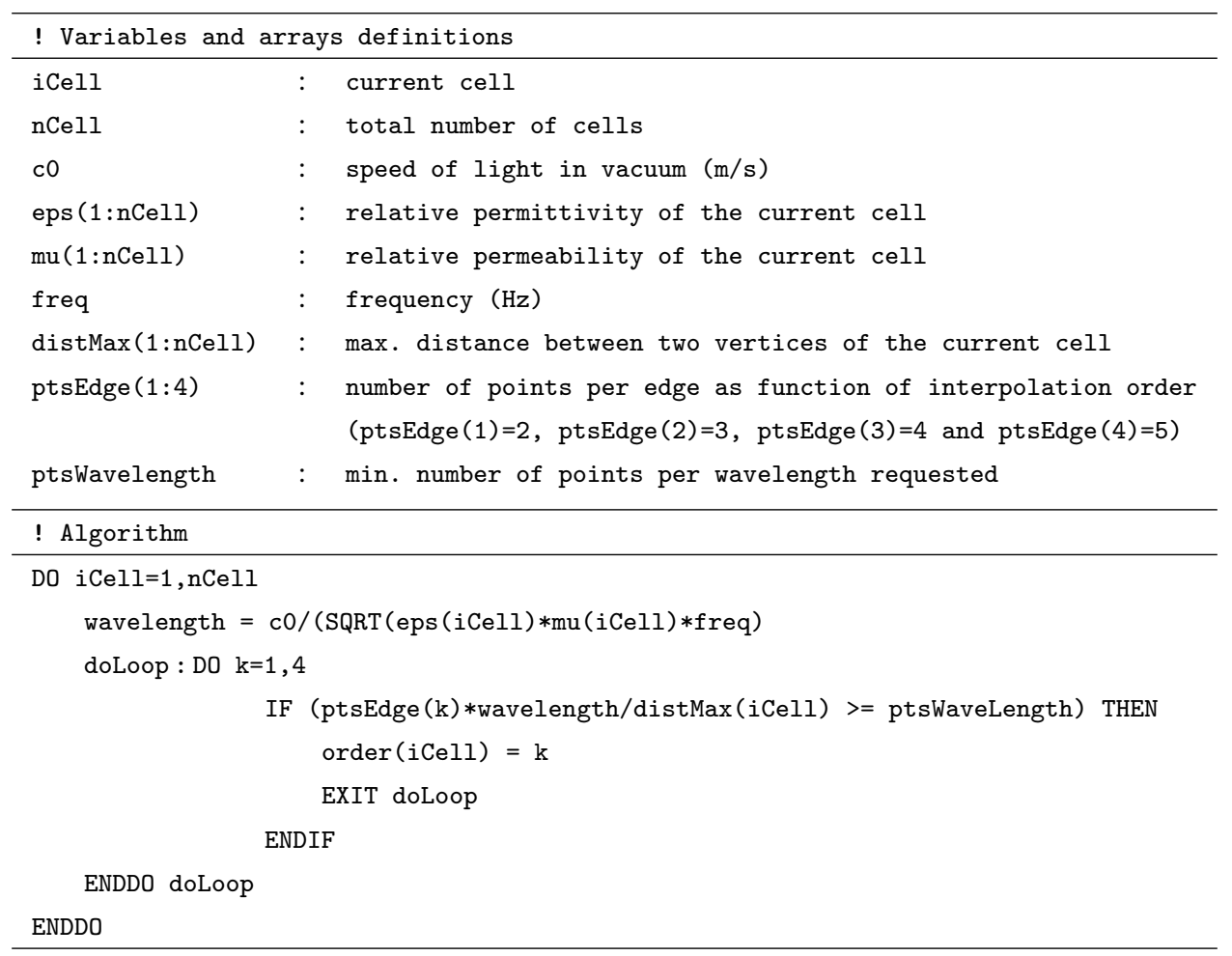

TABLE 14 Exposure of head tissues to a localized source radiation: interpolation order distribution algorithm for the HDG- $\mathbb{P}_{\text {local }}$ method.

\begin{tabular}{rrrrrrr}
\hline & \# Elements & \multicolumn{2}{l}{ \# Faces } & \# DoFs EM & \# DoFs $\boldsymbol{1}$ \\
\hline Coarse mesh (M1) & 366,208 & & 733,856 & & $18,010,230$ & $7,537,090$ \\
$\mathbb{P}_{1}$ & 238,975 & $(65 \%)$ & 462,235 & $(63 \%)$ & $5,735,400$ & $2,773,410$ \\
$\mathbb{P}_{2}$ & 54,525 & $(15 \%)$ & 95,390 & $(13 \%)$ & $3,271,500$ & $1,144,680$ \\
$\mathbb{P}_{3}$ & 69,615 & $(19 \%)$ & 166,793 & $(23 \%)$ & $8,353,800$ & $3,335,860$ \\
$\mathbb{P}_{4}$ & 3,093 & $(1 \%)$ & 9,438 & $(1 \%)$ & 649,530 & 283,140 \\
\hline Fine mesh (M2) & $1,853,832$ & & $3,713,424$ & & $49,312,008$ & $24,352,518$ \\
$\mathbb{P}_{1}$ & $1,720,087$ & $(93 \%)$ & $3,368,875$ & $(91 \%)$ & $41,282,088$ & $20,213,250$ \\
$\mathbb{P}_{2}$ & 133,658 & $(7 \%)$ & 344,289 & $(9 \%)$ & $8,019,480$ & $4,131,468$ \\
$\mathbb{P}_{3}$ & 87 & $(0 \%)$ & 260 & $(0 \%)$ & 10,440 & 7,800 \\
$\mathbb{P}_{4}$ & 0 & $(0 \%)$ & 0 & $(0 \%)$ & 0 & 0 \\
\hline
\end{tabular}

TABLE 15 Exposure of head tissues to a localized source radiation: inerpolation order distribution for the HDG- $\mathbb{P}_{\text {local }}$ method with a minimum of 9 points per wavelength. 

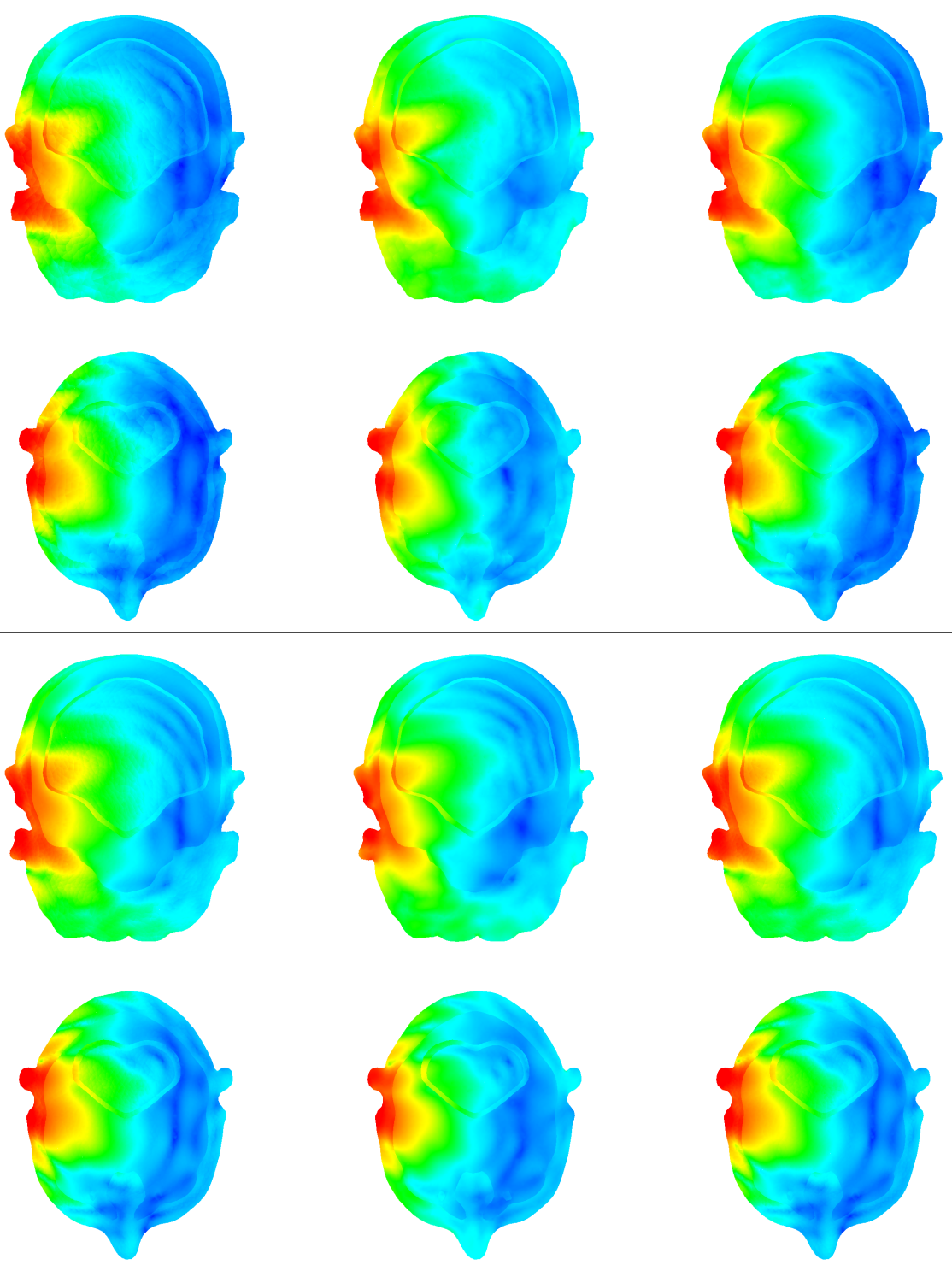

FIGURE 9 Exposure of head tissues to a localized source radiation: contour lines of the local SAR normalized by the maximal local SAR (logarithmic scale) in selected planes for the coarse mesh (top) and the fine mesh (bottom) - From left to right HDG- $\mathbb{P}_{1}$, HDG- $\mathbb{P}_{2}$ and HDG- $\mathbb{P}_{\text {local }}$ results.

problems such as the second problem considered here. Finally, a weak scalability analysis will allow to assess the largest problems that can be solved with the proposed methodology. 


\section{ACKNOWLEDGMENTS}

The work presented here has been financially supported by the PRACE project funded in part by the EU's Horizon 2020 research and innovation programme (2014-2020) under grant agreements 653838 (PRACE 4IP project) and 730913 (PRACE 5IP project), and by the TECSER project funded by ANR (French National Research Agency) under grant agreement ANR-13-ASTR-0027. We also acknowledge GENCI (Big national equipment intensive computing) for granting us access to the Occigen Bull/Atos cluster at CINES. Finally, we are grateful to MUMPS and PaStiX development teams for their precious help to the achievement of this work.

\section{References}

1. Nedelec J. A new family of mixed finite elements in $\mathbb{R}^{3}$. Numer. Math. 1986; 50:57-81.

2. Dolean V, Lanteri S, Perrussel R. A domain decomposition method for solving the three-dimensional time-harmonic Maxwell equations discretized by discontinuous Galerkin methods. J. Comput. Phys. 2007; 227(3): 2044-2072.

3. Dolean V, Fol H, Lanteri S, Perrussel R. Solution of the time-harmonic Maxwell equations using discontinuous Galerkin methods. J. Comput. Appl. Math. 2008; 218: 435-445.

4. Cockburn B, Gopalakrishnan J, Lazarov R. Unified hybridization of discontinuous Galerkin, mixed, and continuous Galerkin methods for second order elliptic problems. SIAM J. Numer. Anal. 2009; 47(2): 1319-1365.

5. Li L, Lanteri S, Perrussel R. Numerical investigation of a high order hybridizable discontinuous Galerkin method for $2 \mathrm{~d}$ timeharmonic Maxwell's equations. COMPEL 2013; 32(3): 1112-1138.

6. Nguyen N, Peraire J, Cockburn B. Hybridizable discontinuous Galerkin methods for the time-harmonic Maxwell's equations. J. Comput. Phys. 2011; 230(19): 7151-7175.

7. Fen X, Lu P, Xu X. A hybridizable discontinuous Galerkin method for the time-harmonic Maxwell equations with high wave number. Comput. Methd. Appl. Math. 2016; 16(3): 429-445.

8. Lu P, Chen H, Qiu W. An absolutely stable hp-HDG method for the time-harmonic Maxwell equations with high wave number. Math. Comp. 2017; 86: 1553-1577.

9. Li L, Lanteri S, Perrussel R. A hybridizable discontinuous Galerkin method combined to a Schwarz algorithm for the solution of 3d time-harmonic Maxwell's equations. J. Comput. Phys. 2014; 256: 563-581.

10. Monk P. Finite element methods for Maxwell's equations. Oxford University Press . 2003.

11. Amestoy P, Duff I, L'Excellent JY. Multifrontal parallel distributed symmetric and unsymmetric solvers. Comput. Meth. App. Mech. Engng. 2000; 184: 501-520.

12. Hénon $P$, Ramet $P$, Roman J. PaStiX: a high-performance parallel direct solver for sparse symmetric definite systems. Paral. Comput. 2002; 28: 301-321.

13. Giraud L, Haidar A, Watson L. Parallel scalability study of hybrid preconditioners in three dimensions. Paral. Comput. 2008; 34 : 363-379.

14. Mathew T. Domain Decomposition methods for the numerical solution of partial differential equations. Springer Lecture Notes in Computational Science and EngineeringSpringer . 2008. 
15. Agullo E, Giraud L, Nakov S, Roman J. Hierarchical hybrid sparse linear solver for multicore platforms. Research Report RR8960, INRIA; : 2016.

16. Després B, Joly P, Roberts J. A domain decomposition method for the harmonic Maxwell equations. In: North-Holland; 1992; Amsterdam: 475-484.

17. Dolean V, Gander M, Gerardo-Giorda L. Optimized Schwarz methods for Maxwell's equations. SIAM J. Scient. Comp. 2009; 31(3): 2193-2213.

18. Magoulès F, Roux F, Salmon S. Optimal discrete transmission conditions for a nonoveralpping domain decomposition method for the Helmholtz equation. SIAM J. Sci. Comput. 2004; 25(5): 1497-1515.

19. Viquerat J, Scheid C. A 3D curvilinear discontinuous Galerkin time-domain solver for nanoscale light-matter interactions. J. Comp. Appl. Math. 2015; 289: 37-50.

20. Bernardi P, Cavagnaro M, Pisa S, Piuzzi E. Specific absorption rate and temperature increases in the head of a cellular phone user. IEEE Trans. Microw. Theor. Tech. 2000; 48(7): 1118-1126.

21. Bernardi P, Cavagnaro M, Pisa S, Piuzzi E. Power absorption and temperature elevations induced in the human head by a dualband monopole-helix antenna phone. IEEE Trans. Microw. Theor. Tech. 2001; 49(12): 2539-2546.

22. Gandhi O, Li QX, Kang G. Temperature rise for the human head for cellular telephones and for peak SARs prescribed in safety guidelines. IEEE Trans. Microw. Theor. Tech. 2001; 49(9): 1607-1613. 